\title{
EXACT CONTROLLABILITY TO TRAJECTORIES FOR SEMILINEAR HEAT EQUATIONS WITH DISCONTINUOUS DIFFUSION COEFFICIENTS
}

\author{
Anna Doubova ${ }^{1}$, A. Osses ${ }^{2}$ And J.-P. Puel ${ }^{3}$
}

\begin{abstract}
The results of this paper concern exact controllability to the trajectories for a coupled system of semilinear heat equations. We have transmission conditions on the interface and Dirichlet boundary conditions at the external part of the boundary so that the system can be viewed as a single equation with discontinuous coefficients in the principal part. Exact controllability to the trajectories is proved when we consider distributed controls supported in the part of the domain where the diffusion coefficient is the smaller and if the nonlinear term $f(y)$ grows slower than $|y| \log ^{3 / 2}(1+|y|)$ at infinity. In the proof we use null controllability results for the associate linear system and global Carleman estimates with explicit bounds or combinations of several of these estimates. In order to treat the terms appearing on the interface, we have to construct specific weight functions depending on geometry.
\end{abstract}

Mathematics Subject Classification. 35B37.

Received October 23, 2001. Revised February 7, 2002.

\section{INTRODUCTION AND HYPOTHESIS}

Let $\Omega \subset \mathbb{R}^{N}, N \geq 1$ be a bounded connected open set with boundary $\Gamma$ of class $C^{2}$. Let $\omega \subset \Omega$ be a nonempty open subset and $T>0$. We will use the following notation: $Q=\Omega \times(0, T), \Sigma=\Gamma \times(0, T)$. For any $p \in[1,+\infty]$, we will denote by $\|\cdot\|_{p}$ the usual norm in $L^{p}(Q)$.

There are two different situations that will be analyzed in this paper. More precisely, let $\Omega_{0}$ and $\Omega_{1}$ be a partition of $\Omega$ in two non empty open sets such that

Case 1: $\quad \bar{\Omega}_{0} \subset \subset \Omega, \quad \Omega_{1}=\Omega \backslash \bar{\Omega}_{0} \quad$ (see Fig. 1, left);

Case 2: $\bar{\Omega}_{1} \subset \subset \Omega, \quad \Omega_{0}=\Omega \backslash \bar{\Omega}_{1} \quad$ (see Fig. 1, right).

Keywords and phrases: Carleman inequalities, controllability, transmission problems.

${ }^{1}$ Departamento E.D.A.N., Universidad de Sevilla, Tarfia s/n, 41012 Sevilla, Spain and École Polytechnique, 91128 Palaiseau Cedex, France; e-mail: dubova@numer.us.es, doubova@cmapx.polytechnique.fr

This work has been partially supported by D.G.E.S., Spain, Grants PB98-1134.

2 Departamento de Ingenería Matemática, Facultad de Ciencias de Físicas y Matemáticas, Universidad de Chile, Casilla 170/3 Correo 3, Santiago, Chile and Centro de Modelamiento Matemático, UMR 2071 CNRS-Uchile; e-mail: axosses@dim.uchile.cl This work has been partially supported by FONDECYT grants No. 1000955 and 7000955.

${ }^{3}$ Laboratoire de Mathématiques Appliquées, Université de Versailles Saint-Quentin, 45 avenue des États Unis, 78035 Versailles Cedex, France and École Polytechnique, 91128 Palaiseau Cedex, France; e-mail: jppuel@cmapx.polytechnique.fr 

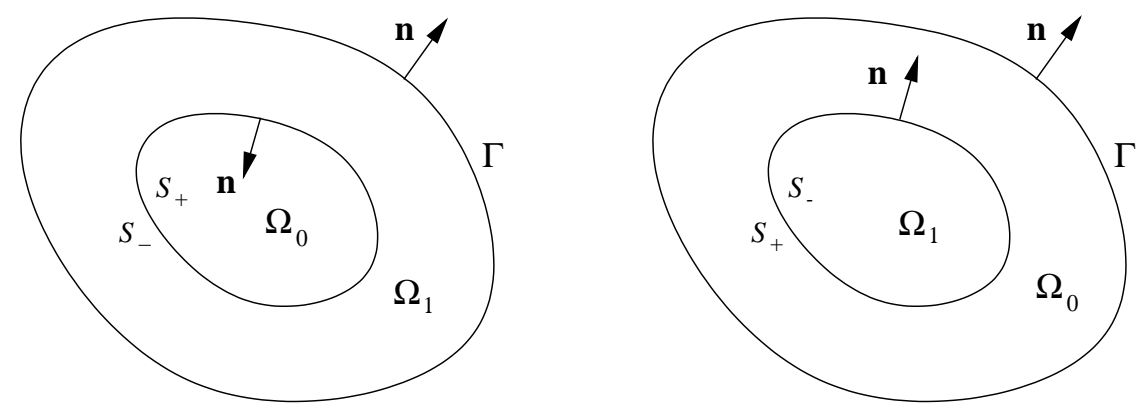

Figure 1. Two geometrical cases covered in this paper depending on $\Omega_{0} \subset \subset \Omega$ or $\Omega_{1} \subset \subset \Omega$.

We denote by $S=\bar{\Omega}_{0} \cap \bar{\Omega}_{1}$ the interface, which will be supposed of class $C^{2}$ and by $n$ the outward unit normal to $\Omega_{1}$ at the points of $S$ and also the outward unit normal to $\Omega$ at the points of $\Gamma$. Let $S^{+}$(resp. $S^{-}$) be the part of $S$ corresponding to the positive (resp. negative) direction of the normal $n$.

Remark 1.1. The two cases mentioned above are not exhaustive, we do not treat other possible geometrical situations in this paper.

In both cases mentioned above, we will consider the following transmission problem for semilinear heat equation

$$
\begin{cases}\partial_{t} y-\operatorname{div}\left(a_{0}(x) \nabla y\right)+f(y)=v 1_{\omega}+g_{0} & \text { in } \quad \Omega_{0} \times(0, T), \\ \partial_{t} y-\operatorname{div}\left(a_{1}(x) \nabla y\right)+f(y)=v 1_{\omega}+g_{1} & \text { in } \Omega_{1} \times(0, T), \\ \left.y\right|_{S^{+} \times(0, T)}=\left.y\right|_{S^{-} \times(0, T)}, & \\ \left.a_{0}(x) \partial_{n} y\right|_{S^{+} \times(0, T)}=\left.a_{1}(x) \partial_{n} y\right|_{S^{-} \times(0, T)}, & \\ y=0, & \text { on } \Sigma \\ y(x, 0)=y_{0} & \text { in } \Omega .\end{cases}
$$

Here $f: \mathbb{R} \rightarrow \mathbb{R}$ is a locally Lipschitz-continuous function, $\partial_{n} y$ denotes the outward normal derivative to $\Omega_{1}$, $y_{0} \in L^{2}(\Omega)$ and $v \in L^{r}\left(0, T ; L^{r}(\omega)\right), g_{i} \in L^{r}\left(0, T ; L^{r}\left(\Omega_{i}\right)\right), i=0,1$ with $r$ such that

$$
\begin{cases}\frac{1}{r}+\frac{N}{2 r}<1 & \text { if } N \geq 2 \\ r=2 & \text { if } N=1\end{cases}
$$

Remark 1.2. We could in fact consider $v \in L^{p}\left(0, T ; L^{q}(\omega)\right), g_{i} \in L^{p}\left(0, T ; L^{q}\left(\Omega_{i}\right)\right), i=0,1$ with $1 / p+N /(2 q)$ $<1$ in order to have $L^{\infty}$ solutions, but in the sake of simplicity we take $p=q=r$.

Remark 1.3. Without loss of generality we can assume $y_{0} \in L^{\infty}(\Omega)$. Otherwise, taking $v=0$ for $t \in(0, \delta)$, $\delta>0$ and thanks to the regularizing effect of parabolic equations, $y(\delta) \in L^{\infty}(\Omega)$ for some $\delta>0[21,22]$.

In $(3), y=y(x, t)$ is the state and $v=v(x, t)$ is the control which acts on the system through $\omega$ since $1_{\omega}$ is the characteristic function of the set $\omega$.

We will assume that the diffusion coefficient in (3) satisfies the following:

$$
\begin{aligned}
& a_{i} \in C^{2}\left(\bar{\Omega}_{i}\right) \quad \text { for } i=0,1, \\
& \left.a_{0}\right|_{S^{+}} \neq\left. a_{1}\right|_{S^{-}} .
\end{aligned}
$$

System (3) represents the coupling between two parabolic semilinear equations whose diffusion coefficient has a jump. At the interface $S$, we impose the continuity of the solution $y$ and also of the fluxes. 
Let us set

$$
a(x)=\left\{\begin{array}{lll}
a_{0}(x) & \text { if } & x \in \Omega_{0} \\
a_{1}(x) & \text { if } & x \in \Omega_{1} .
\end{array}\right.
$$

We also set

$$
g(x)=\left\{\begin{array}{lll}
g_{0}(x) & \text { if } & x \in \Omega_{0} \\
g_{1}(x) & \text { if } & x \in \Omega_{1}
\end{array}\right.
$$

Taking into account notations (6) and (7), problem (3) can be written in the divergence form (with discontinuous diffusion coefficients) as follows:

$$
\begin{cases}\partial_{t} y-\operatorname{div}(a(x) \nabla y)+f(y)=v 1_{\omega}+g & \text { in } Q \\ y=0 & \text { on } \Sigma \\ y(x, 0)=y_{0} & \text { in } \Omega .\end{cases}
$$

We will require $a$ to satisfy

$$
a(x) \geq \alpha>0 \quad \text { a.e. in } \Omega
$$

and the following additional hypothesis:

$$
\left.a_{0}\right|_{S^{+}} \leq\left. a_{1}\right|_{S^{-}}
$$

We assume that for each $\eta>0$, there exists $C_{\eta}>0$ such that

$$
\left|\frac{f(s)-f\left(s^{\prime}\right)}{s-s^{\prime}}\right|^{2 / 3} \leq C_{\eta}+\eta \log \left(1+\left|s-s^{\prime}\right|\right) \quad \forall s, s^{\prime} \in \mathbb{R} .
$$

Let us also consider an "ideal" trajectory $y^{*}$, solution of the problem (without control)

$$
\begin{cases}\partial_{t} y^{*}-\operatorname{div}\left(a(x) \nabla y^{*}\right)+f\left(y^{*}\right)=g & \text { in } Q \\ y^{*}=0 & \text { on } \Sigma \\ y(x, 0)^{*}=y_{0}^{*} & \text { in } \Omega\end{cases}
$$

where $y_{0}^{*} \in L^{2}(\Omega)$ and $g \in L^{r}\left(0, T ; L^{r}(\Omega)\right)$, with $r$ as in (4). We know that under conditions (9) and (11), problem (12) possesses exactly one local solution in time ( $c f$. [21] and [22]). Moreover, we can say that there exists a time $T^{*}>0$, such that for $T<T^{*}$, the solution $y^{*}$ of $(12)$ satisfies $y^{*} \in C^{0}\left([0, T] ; L^{2}(\Omega)\right) \cap L^{\infty}\left(\delta, T ; L^{\infty}(\Omega)\right)$, for every $\delta>0$.

The main goal of this paper is to analyze the controllability properties of (8).

Definition 1.1. We say that (8) is exactly controllable to the trajectories if, for any trajectory $y^{*}$ solution of (12) and for any initial condition $y_{0} \in L^{2}(\Omega)$, for every $T<T^{*}$, there exists a control $v \in L^{r}\left(0, T ; L^{r}(\omega)\right)$ such that $(8)$ has a solution $y$ on $(0, T)$ satisfying

$$
y(x, T)=y^{*}(x, T) \quad \text { in } \Omega .
$$


Definition 1.2. System (8) is said null controllable at time $T$ if, for each $y_{0} \in L^{2}(\Omega)$, there exists $v \in$ $L^{r}\left(0, T ; L^{r}(\omega)\right)$ such that the corresponding initial boundary problem $(8)$ admits a solution $y \in C^{0}\left([0, T] ; L^{2}(\Omega)\right)$ satisfying

$$
y(x, T)=0 \quad \text { in } \Omega .
$$

For linear problems, it is easy to see that the notions of null controllability and exact controllability to the trajectories are equivalent, but this is not true for nonlinear systems.

Definition 1.3. It will be said that (8) is approximately controllable in $L^{2}(\Omega)$ at time $T$ if, for any $y_{0} \in L^{2}(\Omega)$, any $y_{d} \in L^{2}(\Omega)$ and any $\varepsilon>0$, there exists a control $v \in L^{r}\left(0, T ; L^{r}(\omega)\right)$ such that the corresponding initial boundary problem (8) possesses a solution $y \in C^{0}\left([0, T] ; L^{2}(\Omega)\right)$, with

$$
\left\|y(\cdot, T)-y_{d}\right\|_{L^{2}(\Omega)} \leq \varepsilon .
$$

In the case in which the diffusion coefficients are sufficiently regular, the controllability of linear and semilinear parabolic systems has been analyzed in several recent papers. Among them, let us mention [1,5,11,13,15-17], and [8] concerning null controllability $[9,12,13,25]$ and [8] for approximate controllability [16] and [13] for exact controllability to the trajectories.

\section{MAIN RESUlt}

\subsection{Geometric hypothesis and main result}

In order to state the main result of this work, we need the following geometrical conditions.

Condition 2.1 (corresponding to case (1)). We assume that there exists a vector field $\zeta: \bar{\Omega}_{1} \mapsto \mathbb{R}^{N}, \zeta \in$ $C^{1}\left(\bar{\Omega}_{1}\right)$, such that

$$
\begin{aligned}
\zeta(x) \cdot n(x)<0 & \forall x \in \Gamma \\
\zeta(x) \cdot n(x)>0 & \forall x \in S, \\
\zeta(x) \neq 0 & \forall x \in \Omega_{1}
\end{aligned}
$$

and if we consider the characteristics associated to $\zeta$

$$
\left\{\begin{array}{l}
\frac{\mathrm{d} x(t)}{\mathrm{d} t}=\zeta(x(t)), \quad t>0, \\
x(0)=x_{0}
\end{array}\right.
$$

with $x_{0} \in \Gamma$, we also assume that for some time $T_{1}>0$ and for every $x_{0} \in \Gamma$, there exists $t_{1}\left(x_{0}\right)<T_{1}$ such that the solution $x(t)$ of (19) verifies

$$
x(t) \in \Omega_{1} \quad \text { for } 0<t<t_{1}\left(x_{0}\right)
$$

and

$$
x\left(t_{1}\left(x_{0}\right)\right) \in S \quad \text { for } x_{0} \in \Gamma .
$$

Remark 2.1. Condition 2.1 implies that $\Gamma$ and $S$ are isotopic, but it is not clear whether isotopy is sufficient to ensure this condition.

Remark 2.2. Notice that Condition 2.1 is fulfilled for usual domains, see for example the cases of Figure 2. 


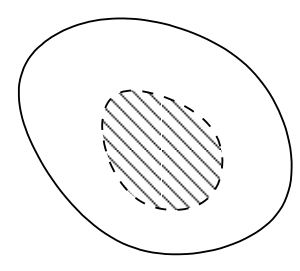

(a)

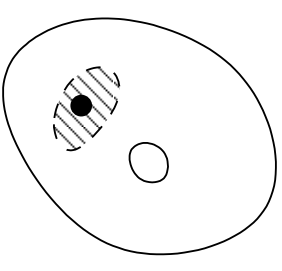

(b)

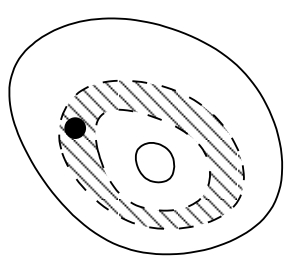

(c)

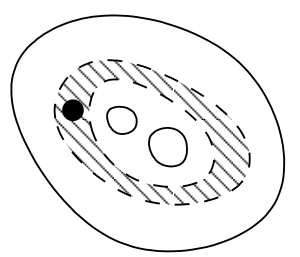

(d)

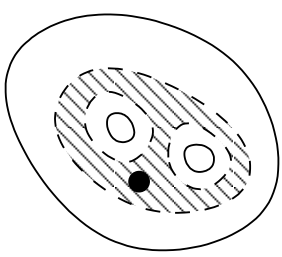

(e)

Figure 2. Condition 2.1 is fulfilled in situations (a, c, e) but not in (b) and (d). The boundary $\Gamma$ is represented by a solid line and the interface $S$ by a dashed line, the dashed region represents $\Omega_{0}$ and the black dot the location of the control zone.

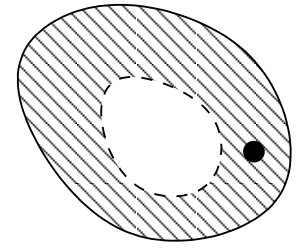

(a)

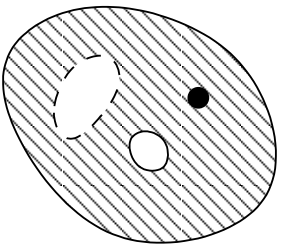

(b)

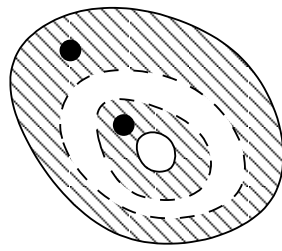

(c)

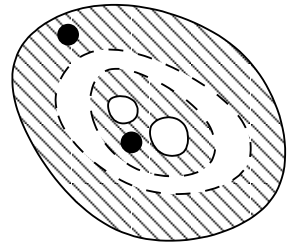

(d)

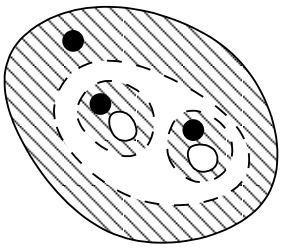

(e)

Figure 3. Condition 2.2 is fulfilled in situations $(\mathrm{a}-\mathrm{d})$ but not in (e) with the same notations as in the previous figure.

Condition 2.2 (corresponding to case (2)). We assume that there exist two disjoint open sets $\mathcal{O}_{1}, \mathcal{O}_{2} \subset \subset \Omega_{1}$ (with always a unit outward normal $n$ ) and vectors fields $\xi^{i}: \bar{\Omega}_{1} \mapsto \mathbb{R}^{N}, \xi^{i} \in C^{1}\left(\bar{\Omega}_{1}\right), i=1,2$, such that

$$
\begin{array}{ll}
\xi^{i}(x) \cdot n(x)>0 & \forall x \in S, \\
\xi^{i}(x) \cdot n(x)>0 & \forall x \in \partial \mathcal{O}_{i}, \quad i=1,2, \\
\xi^{i}(x) \neq 0 & \forall x \in \Omega_{1} \backslash \mathcal{O}_{i}
\end{array}
$$

and for the characteristics associated to $\xi^{i}$

$$
\left\{\begin{array}{l}
\frac{\mathrm{d} x^{i}(t)}{\mathrm{d} t}=-\xi^{i}\left(x^{i}(t)\right), \quad t>0 \\
x^{i}(0)=x_{0}^{i}
\end{array}\right.
$$

with $x_{0}^{i} \in S$, we assume also that for some time $T_{2}^{i}>0$, and for all $x_{0}^{i} \in S$, there exists $t_{2}^{i}\left(x_{0}^{i}\right)<T_{2}^{i}$ such that the solution $x^{i}(t)$ of $(23)$ verifies

$$
x^{i}(t) \in \Omega_{1} \backslash \mathcal{O}_{i} \quad \text { for } 0<t<t_{2}^{i}\left(x_{0}^{i}\right)
$$

and

$$
x^{i}\left(t_{2}^{i}\left(x_{0}^{i}\right)\right) \in \partial \mathcal{O}_{i} \quad \text { for } x_{0}^{i} \in S, i=1,2
$$

Remark 2.3. Notice that this hypothesis is essentially Condition 2.1 written for the case (2). It is also fulfilled in usual geometrical cases, see for example the cases in Figure 3. 
The aim of this paper is to prove the following theorem:

Theorem 2.1. Assume that in problem (8) the coefficient a satisfies (5, 6, 9, 10), $f$ is a locally Lipschitzcontinuous function satisfying (11) or Condition 2.1 in case (1) or Condition 2.2 in case (2) are fulfilled. If $\omega \cap \Omega_{0}^{i} \neq \emptyset$, for each connected component $\Omega_{0}^{i}$ of $\Omega_{0}$, then for each case (1) or (2, 8) is exactly controllable to the trajectories.

The idea of the proof of Theorem 2.1 is the following. With a simple change of variables we reduce the problem of exact controllability to the trajectories for (8) to null controllability for a still nonlinear similar transmission problem. For this null controllability result we use approximate controllability to the zero state for an associated linear transmission problem with controls in $L^{r}\left(0, T ; L^{r}(\omega)\right)$ for $r$ as in (4) and then we apply a fixed point method. For this we need explicit estimates on the cost of approximate controllability which is obtained from observability inequalities (see Props. 4.1 and 4.2). These estimates are deduced from global Carleman inequalities. In case (1), we use one single global Carleman inequality (see Th. 3.3) with a suitable weight function, whose construction is presented in Lemma 3.1. Case (2) is more complicated and we have to combine two different global Carleman inequalities (see Th. 3.4) with two appropriate weight functions whose construction are given in Lemma 3.2. The growth condition of the non linear term $f$ is analyzed using the arguments of [13].

The idea of combining the controllability of a linearized system and a fixed point argument in the proof is rather general. It was introduced in [23] in the context of the boundary controllability of the semilinear wave equation. For other controllability results proved in a similar way, see for instance $[9,13,15]$ and $[8]$.

In the proofs we will suppose that $\Omega_{0}$ and $\Omega_{1}$ are connected sets and we assume the simpler hypothesis $\omega \cap \Omega_{0} \neq \emptyset$. Otherwise the weight functions for Carleman inequalities are constructed analogously on each connected component of $\Omega_{0}$ and $\Omega_{1}$.

The paper is organized as follows. In Section 3 we deduce global Carleman inequalities, that we use for proving the main result. Section 4 is devoted to obtain some observability estimates. In Section 5 , we prove Theorem 2.1. Finally, in Section 6, we give an explicit construction of suitable weight functions, needed for the global Carleman inequalities.

\subsection{Some consequences and extensions}

1. Observe that, the controllability result holds if the control acts in the part of the domain where the diffusion coefficient is smaller. To our knowledge, this result is the first one in the literature related to exact controllability to the trajectories when the diffusion coefficients are discontinuous.

2. In the case $s^{\prime}=0$ and $f(0)=0$, notice that assumption (11) can be simply read as follows:

$$
\lim _{|s| \rightarrow+\infty} \frac{f(s)}{|s| \log ^{3 / 2}(1+|s|)}=0 .
$$

The proof of Theorem 2.1 also gives the result of null controllability for (8) with a suitable hypothesis on $g$ under the hypothesis and the same geometrical cases considered in Theorem 2.1 by taking condition (24) instead of (11).

3. Notice that approximate controllability for a linear transmission problem is always true and it is independent of the choice of the part of the domain where the control acts as a consequence of unique continuation property. Nonlinear problem (8) with $f$ growing as in (11) is still approximately controllable under the conditions of Theorem 2.1. This is due to the fact that approximate controllability in this case can be proved as a consequence of exact controllability to the trajectories. This idea is taken from [12], where approximate controllability for semilinear heat equations is obtained in such a way. 
4. We can also consider in (8) the more general case in which the diffusion coefficients are represented by a real symmetric uniformly elliptic matrix $A$, i.e. there exists a constant $\alpha>0$ such that

$$
A(x, \xi, \xi)=\sum_{i, j=1}^{N} A_{i j} \xi_{i} \xi_{j} \geq \alpha|\xi|^{2} \quad \forall \xi \in \mathbb{R}^{N}, \text { for a.e. } x \in \Omega
$$

and $A$ is regular in each $\Omega_{i}, i=0,1$. In this case, condition (10) has to be replaced by

$$
\left[\frac{\operatorname{det} A}{A n \cdot n}\right]_{S} \geq 0
$$

where []$_{S}$ denotes the jump across $S$.

Until now, null controllability for semilinear parabolic systems (in the divergence form) has been analyzed when the diffusion coefficients are sufficiently regular. More precisely, when $A=\left(A_{i j}\right), i, j=1, \ldots, N$ with $A_{i j} \in C^{1,2}(\bar{Q})($ see $[15])$.

\subsection{Open problems related to Theorem 2.1}

1. If $\omega \subset \Omega_{1}$ we do not know whether or not system (8) is exactly controllable to the trajectories. Is in this case null controllability also an open problem.

2. In [13], it is proved that even in the case of regular diffusion coefficients, for each $\beta>2$, there exist functions $f=f(s)$ with $f(0)=0$ and

$$
\lim _{|s| \rightarrow \infty} \frac{|f(s)|}{|s| \log ^{\beta}(1+|s|)}=\alpha \quad \text { with } \alpha>0
$$

such that the corresponding system (for the semilinear heat equation) is not null-controllable for any $T>0$. In view of point 2 in Section 2.2 , we see that, when $f$ satisfies (27) with $3 / 2 \leq \beta \leq 2$, null-controllability of (8) is an open question.

3. On the other hand, it is proved in [13], that also in the case of regular diffusion coefficients, for each $\beta>2$, there exist functions $f$ satisfying (27) such that the corresponding system (for the semilinear heat equation) is not approximately controllable for all $T>0$. Then, approximate controllability for the transmission problem (8) with $3 / 2 \leq \beta \leq 2$, is also an open question.

4. An abstract result due to Russell [20] shows that boundary exact controllability for the wave equation implies boundary exact null controllability for the heat equation with the same type of control and geometry. This result is proved in the case of smooth coefficients. If we consider this principle still true in the case of non smooth coefficients, the geometrical hypothesis that we consider here seems to be too restrictive in the case $N=1$ ( $c f .[7]$ for the controllability of the corresponding wave equation) but not for $N \geq 2$.

5. In [14], it is proved null controllability result for the one-dimensional linear heat equation like $\rho(x)-$ $\left(a(x) z_{x}\right)_{x}+m(x) z=0$ with only BV coefficients without any assumption on the control zone. However, the proof is definitely strictly one dimensional relying on the corresponding one for the wave equation and null controllability result is true if the potential $m$ depends only on space variable, but not on time. Then, even in the one-dimensional case it is not clear how to treat with this method a similar nonlinear problem. 


\section{Global Carleman inequalities}

In this section we will deduce two global Carleman inequalities, that we need for the proof of Theorem 2.1. For this purpose, we will introduce suitable weight functions. Let us first consider the situation of case (1) (see Fig. 1, left). The first weight function is given by the following result:

Lemma 3.1. Assume that we have the geometrical situation of case (1) (see Fig. 1). Assume that the function a defined in $(5,6)$ satisfies $(9,10)$ and that Condition 2.1 holds. If $\omega \cap \Omega_{0} \neq \emptyset$ then for every open set $\omega_{0} \subset \subset \omega \cap \Omega_{0}$ there exists a function $\widetilde{\beta} \in C^{0}(\bar{\Omega}), \widetilde{\beta}_{i}=\left.\widetilde{\beta}\right|_{\Omega_{i}} \in C^{2}\left(\bar{\Omega}_{i}\right), i=0,1, \widetilde{\beta}>0$ in $\Omega$, such that

$$
\begin{array}{rc}
\widetilde{\beta}=0 & \text { on } \Gamma, \\
\partial_{n} \widetilde{\beta}<0 & \text { on } \Gamma, \\
\widetilde{\beta}=1 & \text { on } S, \\
\partial_{n} \widetilde{\beta}_{0}>0, \partial_{n} \widetilde{\beta}_{1}>0 & \text { on } S, \\
a_{0} \partial_{n} \widetilde{\beta}_{0}=a_{1} \partial_{n} \widetilde{\beta}_{1} & \text { on } S
\end{array}
$$

and

$$
|\nabla \widetilde{\beta}|>0 \quad \text { in } \bar{\Omega} \backslash \omega_{0} .
$$

The proof of Lemma 3.1 will be given in Section 6 .

Now, we consider the geometrical case (2) (see Fig. 1, right). For the second Carleman inequality, which we will use to treat the situation 2 , we need two suitable weight functions.

We have the following result:

Lemma 3.2. Assume that we have the geometrical situation of case (2) (see Fig. 1). Assume that the function a defined in $(5,6)$ satisfies $(9,10)$ and that there exist two open disjoint sets $\mathcal{O}_{1}, \mathcal{O}_{2} \subset \subset \Omega_{1}$ verifying Condition 2.2. Let $B_{i}$ and $\widetilde{B}_{i}, i=1,2$ be balls such that $B_{1} \subset \subset \widetilde{B}_{1} \subset \subset \mathcal{O}_{1}$ and $B_{2} \subset \subset \widetilde{B}_{2} \subset \subset \mathcal{O}_{2}$. If $\omega \cap \Omega_{0} \neq \emptyset$ then for every open set $\omega_{0} \subset \subset \omega \cap \Omega_{0}$ there exist two functions $\widetilde{\beta}^{1}$ and $\widetilde{\beta}^{2}$ such that

$$
\widetilde{\beta}^{1}(x)=\left\{\begin{array}{ll}
\widetilde{\beta}_{0}(x) & \text { if } \quad x \in \Omega_{0}, \\
\widetilde{\beta}_{1}^{1}(x) & \text { if } \quad x \in \Omega_{1},
\end{array} \quad \widetilde{\beta}^{2}(x)=\left\{\begin{array}{lll}
\widetilde{\beta}_{0}(x) & \text { if } & x \in \Omega_{0}, \\
\widetilde{\beta}_{1}^{2}(x) & \text { if } & x \in \Omega_{1},
\end{array}\right.\right.
$$

with the following properties: $\widetilde{\beta}_{0} \in C^{2}\left(\bar{\Omega}_{0}\right), \widetilde{\beta}_{0}>0$ in $\Omega_{0}$,

$$
\begin{gathered}
\widetilde{\beta}_{0}=0 \text { on } \Gamma, \quad \partial_{n} \widetilde{\beta}_{0}<0 \text { on } \Gamma, \\
\partial_{n} \widetilde{\beta}_{0}>0 \text { on } S, \quad \widetilde{\beta}_{0}=2 \text { on } S, \\
\left|\nabla \widetilde{\beta}_{0}\right|>0 \quad \text { in } \quad \bar{\Omega}_{0} \backslash \omega_{0} .
\end{gathered}
$$

And for $i=1,2, \widetilde{\beta}_{1}^{i} \in C^{2}\left(\bar{\Omega}_{1}\right), \widetilde{\beta}_{1}^{i}>0$ in $\Omega_{1}$,

$$
\begin{aligned}
\widetilde{\beta}_{1}^{i}=\widetilde{\beta}_{0}=2 & \text { on } S, \\
a_{0} \partial_{n} \widetilde{\beta}_{0}=a_{1} \partial_{n} \widetilde{\beta}_{1}^{i} & \text { on } S, \quad i=1,2, \\
\widetilde{\beta}_{1}^{1} \geq 2 \widetilde{\beta}_{1}^{2} & \text { in } \widetilde{B}_{2}, \\
\widetilde{\beta}_{1}^{2} \geq 2 \widetilde{\beta}_{1}^{1} & \text { in } \widetilde{B}_{1},
\end{aligned}
$$


and

$$
\left|\nabla \widetilde{\beta}_{1}^{i}\right|>0 \quad \text { in } \bar{\Omega}_{1} \backslash B_{i}, \quad i=1,2 .
$$

The proof of Lemma 3.2 will also be given in Section 6 .

Remark 3.1. Notice that in geometrical case $(2)\left(\Omega_{1} \subset \subset \Omega\right)$ it is impossible to have a function $\widetilde{\beta}$ which is constant on $S$ and such that $\nabla \widetilde{\beta} \neq 0$ in $\Omega_{1}$.

Let us consider the functions

$$
\beta=\widetilde{\beta}+K, \quad \bar{\beta}=\frac{5}{4} \max _{\bar{\Omega}} \beta,
$$

with $K>0$ such that $K \geq 5 \max _{\bar{\Omega}} \widetilde{\beta}$, and $\widetilde{\beta}$ is given by Lemma 3.1 .

Let $\lambda$ be a sufficiently large positive constant that only depends on $\Omega$ and $\omega$. It will be fixed later on. For $t \in(0, T)$ and following [16] and [12], we introduce the following functions:

$$
\varphi(x, t)=\frac{\mathrm{e}^{\lambda \beta(x)}}{t(T-t)}, \quad \eta(x, t)=\frac{\mathrm{e}^{\lambda \bar{\beta}}-\mathrm{e}^{\lambda \beta}}{t(T-t)} .
$$

Notice that

$$
\nabla \eta=-\lambda \varphi \nabla \beta, \quad \nabla \varphi=\lambda \varphi \nabla \beta
$$

Let us set

We have the following Carleman estimate:

$$
\begin{array}{r}
Z_{0}=\left\{q: q \in C^{2}\left(\bar{\Omega}_{i} \times[0, T]\right), \quad i=0,1,\left.\quad q\right|_{S^{+} \times(0, T)}=\left.q\right|_{S^{-} \times(0, T)},\right. \\
\left.\left.a_{0} \partial_{n} q\right|_{S^{+} \times(0, T)}=\left.a_{1} \partial_{n} q\right|_{S^{-} \times(0, T)}, \quad q=0 \quad \text { on } \Sigma\right\} .
\end{array}
$$

Theorem 3.3. Assume that $\omega \cap \Omega_{0} \neq \emptyset$, a satisfies (5, 6, 9) and (10) and Condition 2.1 in case (1) is fulfilled. There exists $\lambda_{1}(\Omega, \omega, a)>0$ such that for each $\lambda>\lambda_{1}$ there exists a positive constant $C$ that only depends on $\Omega$, $\omega$ and $a$, and $s_{1}(\lambda)>0$ so that the following estimate holds

$$
\begin{gathered}
s^{3} \iint_{Q} \mathrm{e}^{-2 s \eta} t^{-3}(T-t)^{-3}|q|^{2} \mathrm{~d} x \mathrm{~d} t+s \iint_{Q} \mathrm{e}^{-2 s \eta} t^{-1}(T-t)^{-1}|\nabla q|^{2} \mathrm{~d} x \mathrm{~d} t \\
\leq C\left(s^{3} \iint_{\omega \times(0, T)} \mathrm{e}^{-2 s \eta} t^{-3}(T-t)^{-3}|q|^{2} \mathrm{~d} x \mathrm{~d} t\right. \\
\left.+\iint_{Q} \mathrm{e}^{-2 s \eta}\left|\partial_{t} q+\operatorname{div}(a(x) \nabla q)\right|^{2} \mathrm{~d} x \mathrm{~d} t\right)
\end{gathered}
$$

for all $q \in Z_{0}$ and $s \geq s_{1}$. Moreover, $s_{1}$ is of the form $s_{1}=\sigma_{1}(\Omega, \omega, a, \lambda)\left(T^{2}+T\right)$, where $\sigma_{1}$ is a positive constant that only depends on $\Omega, \omega$, a and $\lambda$.

Proof of the Theorem 3.3. In the sequel, $C$ will stand for a generic positive constant only depending on $\Omega, \omega$ and $a$, whose value can change from line to line. We will also use the usual convention of repeated indices.

Let us assume $q \in Z_{0}$ and $s>0$. We set

$$
f=\partial_{t} q+\operatorname{div}(a(x) \nabla q)
$$


and

$$
\psi=\mathrm{e}^{-s \eta} q
$$

Notice that

$$
\psi(0)=\psi(T)=0
$$

We have the following equality:

$$
\mathrm{e}^{-s \eta}\left(\partial_{t}\left(\mathrm{e}^{s \eta} \psi\right)+\operatorname{div}\left(a(x) \nabla\left(\mathrm{e}^{s \eta} \psi\right)\right)=\mathrm{e}^{-s \eta} f .\right.
$$

Using (45), we can write (49) in the form

$$
M_{1} \psi+M_{2} \psi=\mathrm{e}^{-s \eta} f+s \lambda \varphi \operatorname{div}(a(x) \nabla \beta) \psi-s \lambda^{2} \varphi a(x)|\nabla \beta|^{2} \psi,
$$

where

$$
M_{1} \psi=\operatorname{div}(a(x) \nabla \psi)+s^{2} \lambda^{2} \varphi^{2}|\nabla \beta|^{2} a(x) \psi+s \partial_{t} \eta \psi
$$

and

$$
M_{2} \psi=\partial_{t} \psi-2 s \lambda \varphi a(x) \nabla \beta \nabla \psi-2 s \lambda^{2} \varphi a(x)|\nabla \beta|^{2} \psi .
$$

Let set

$$
f_{s}=\mathrm{e}^{-s \eta} f+s \lambda \varphi \operatorname{div}(a(x) \nabla \beta) \psi-s \lambda^{2} \varphi a(x)|\nabla \beta|^{2} \psi .
$$

From (50), we obtain

$$
\left\|M_{1} \psi\right\|_{2}^{2}+\left\|M_{2} \psi\right\|_{2}^{2}+2\left(M_{1} \psi, M_{2} \psi\right)=\left\|f_{s}\right\|_{2}^{2},
$$

where $(\cdot, \cdot)$ denotes the scalar product in $L^{2}(Q)$. Let us compute the scalar product in the left hand side of $(54)$. We can write

$$
\left(M_{1} \psi, M_{2} \psi\right)=I_{11^{\prime}}+I_{12^{\prime}}+I_{13^{\prime}}+I_{21^{\prime}}+I_{22^{\prime}}+I_{23^{\prime}}+I_{31^{\prime}}+I_{32^{\prime}}+I_{33^{\prime}} .
$$

In (55), all the integrals denote the respective scalar products for the terms of $M_{1} \psi$ and $M_{2} \psi$. For simplicity, in the sequel, we will write $a$ instead of $a(x)$. We have

$$
\begin{aligned}
I_{11^{\prime}}= & \iint_{Q} \operatorname{div}(a \nabla \psi) \partial_{t} \psi \mathrm{d} x \mathrm{~d} t=-\iint_{Q} a \nabla \psi \partial_{t}(\nabla \psi) \mathrm{d} x \mathrm{~d} t \\
& +\int_{0}^{T} \int_{S} a_{1} \nabla \psi \cdot n \partial_{t} \psi \mathrm{d} x \mathrm{~d} t-\int_{0}^{T} \int_{S} a_{0} \nabla \psi \cdot n \partial_{t} \psi \mathrm{d} x \mathrm{~d} t=-\frac{1}{2} \iint_{Q} a \partial_{t}\left(|\nabla \psi|^{2}\right) \mathrm{d} x \mathrm{~d} t=0 .
\end{aligned}
$$


Here we have used (48) which says that $\psi(0)=\psi(T)=0$.

$$
\begin{aligned}
I_{12^{\prime}}= & -2 s \lambda \sum_{l=0}^{1} \int_{0}^{T} \int_{\Omega_{l}} \varphi \operatorname{div}\left(a_{l} \nabla \psi\right) a_{l} \nabla \beta \nabla \psi \mathrm{d} x \mathrm{~d} t=2 s \lambda \sum_{l=0}^{1} \int_{0}^{T} \int_{\Omega_{l}} a_{l} \partial_{x_{i}} \psi \partial_{x_{i}}\left(\varphi a_{l} \partial_{x_{j}} \beta \partial_{x_{j}} \psi\right) \mathrm{d} x \mathrm{~d} t \\
& -2 s \lambda \int_{0}^{T} \int_{S^{-}} \varphi a_{1}^{2}\left(\nabla \beta_{1} \cdot \nabla \psi\right)(\nabla \psi \cdot n) \mathrm{d} \sigma \mathrm{d} t+2 s \lambda \int_{0}^{T} \int_{S^{+}} \varphi a_{0}^{2}\left(\nabla \beta_{0} \cdot \nabla \psi\right)(\nabla \psi \cdot n) \mathrm{d} \sigma \mathrm{d} t \\
& -2 s \lambda \int_{0}^{T} \int_{\Gamma} \varphi a_{1}^{2}(\nabla \beta \cdot \nabla \psi)(\nabla \psi \cdot n) \mathrm{d} \sigma \mathrm{d} t .
\end{aligned}
$$

Let us consider the first term of (57). Also for simplicity, we will make the computation only for the integrals in $\Omega_{1}$. We set

$$
I_{12^{\prime}}^{1}=2 s \lambda \int_{0}^{T} \int_{\Omega_{1}} a_{1} \partial_{x_{i}} \psi \partial_{x_{i}}\left(\varphi a_{1} \partial_{x_{j}} \beta \partial_{x_{j}} \psi\right) \mathrm{d} x \mathrm{~d} t
$$

We will use (45) and

$$
\begin{aligned}
& \nabla \beta=(\nabla \beta \cdot n) n+\nabla_{\tau} \beta, \\
& \nabla \psi=(\nabla \psi \cdot n) n+\nabla_{\tau} \psi,
\end{aligned}
$$

where $\nabla_{\tau} \beta$ and $\nabla_{\tau} \psi$ denote the tangential gradients. Thanks to the choice of $\beta$ we know from (30) and (43) that $\beta$ is a constant on $S$, then $\nabla_{\tau} \beta=0$ on $S$ and we can write

$$
\begin{aligned}
I_{12^{\prime}}^{1}= & 2 s \lambda^{2} \int_{0}^{T} \int_{\Omega_{1}} \varphi\left(a_{1} \partial_{x_{i}} \psi \partial_{x_{i}} \beta\right)\left(a_{1} \partial_{x_{j}} \beta \partial_{x_{j}} \psi\right) \mathrm{d} x \mathrm{~d} t+2 s \lambda \int_{0}^{T} \int_{\Omega_{1}} \varphi a_{1} \partial_{x_{i}} \psi \partial_{x_{i}}\left(a_{1} \partial_{x_{j}} \beta\right) \partial_{x_{j}} \psi \mathrm{d} x \mathrm{~d} t \\
& +s \lambda \int_{0}^{T} \int_{\Omega_{1}} \varphi a_{1}\left(a_{1} \partial_{x_{j}} \beta\right) \partial_{x_{j}}\left(|\nabla \psi|^{2}\right) \mathrm{d} x \mathrm{~d} t .
\end{aligned}
$$

Integrating now by parts in the third term of (60) we obtain

$$
\begin{aligned}
I_{12^{\prime}}^{1}= & 2 s \lambda^{2} \int_{0}^{T} \int_{\Omega_{1}} \varphi\left(a_{1} \partial_{x_{i}} \psi \partial_{x_{i}} \beta\right)\left(a_{1} \partial_{x_{j}} \beta \partial_{x_{j}} \psi\right) \mathrm{d} x \mathrm{~d} t+2 s \lambda \int_{0}^{T} \int_{\Omega_{1}} \varphi a_{1} \partial_{x_{i}} \psi \partial_{x_{i}}\left(a_{1} \partial_{x_{j}} \beta\right) \partial_{x_{j}} \psi \mathrm{d} x \mathrm{~d} t \\
& -s \lambda^{2} \int_{0}^{T} \int_{\Omega_{1}} \varphi\left|a_{1} \nabla \beta\right|^{2}|\nabla \psi|^{2} \mathrm{~d} x \mathrm{~d} t-s \lambda \int_{0}^{T} \int_{\Omega_{1}} \varphi a_{1} \partial_{x_{j}}\left(a_{1} \partial_{x_{j}} \beta\right)|\nabla \psi|^{2} \mathrm{~d} x \mathrm{~d} t \\
& -s \lambda \int_{0}^{T} \int_{\Omega_{1}} \varphi\left(\partial_{x_{j}} a_{1}\right)\left(a_{1} \partial_{x_{j}} \beta\right)|\nabla \psi|^{2} \mathrm{~d} x \mathrm{~d} t+s \lambda \int_{0}^{T} \int_{S^{-}} \varphi a_{1}^{2}\left(\partial_{n} \beta_{1}\right)|\nabla \psi|^{2} \mathrm{~d} \sigma \mathrm{d} t \\
& +s \lambda \int_{0}^{T} \int_{\Gamma} \varphi a_{1}^{2}\left(\partial_{n} \beta\right)|\nabla \psi|^{2} \mathrm{~d} \sigma \mathrm{d} t .
\end{aligned}
$$

For the integrals in $\Omega_{0}$ it is sufficient to take into account that $n$ is the outward unit normal to $\Omega_{1}$ and replace in (61), $n$ by $-n, S^{-}$by $S^{+}$and $\Omega_{1}$ by $\Omega_{0}$. 
Consequently, from (57) and (61), using again (59) we deduce

$$
\begin{aligned}
I_{12^{\prime}}= & -s \lambda^{2} \iint_{Q} \varphi|a \nabla \beta|^{2}|\nabla \psi|^{2} \mathrm{~d} x \mathrm{~d} t+2 s \lambda^{2} \iint_{Q} \varphi(a \nabla \psi \cdot \nabla \beta)^{2} \mathrm{~d} x \mathrm{~d} t \\
& -s \lambda \int_{0}^{T} \int_{S^{-}} \varphi\left(\partial_{n} \beta_{1}\right)\left|a_{1} \partial_{n} \psi\right|^{2} \mathrm{~d} \sigma \mathrm{d} t+s \lambda \int_{0}^{T} \int_{S^{+}} \varphi\left(\partial_{n} \beta_{0}\right)\left|a_{0} \partial_{n} \psi\right|^{2} \mathrm{~d} \sigma \mathrm{d} t \\
& +s \lambda \int_{0}^{T} \int_{S^{-}} \varphi a_{1}\left(a_{1} \partial_{n} \beta_{1}\right)\left|\nabla_{\tau} \psi\right|^{2} \mathrm{~d} \sigma \mathrm{d} t-s \lambda \int_{0}^{T} \int_{S^{+}} \varphi a_{0}\left(a_{0} \partial_{n} \beta_{0}\right)\left|\nabla_{\tau} \psi\right|^{2} \mathrm{~d} \sigma \mathrm{d} t \\
& -s \lambda \int_{0}^{T} \int_{\Gamma} \varphi a_{1}^{2}\left(\partial_{n} \beta\right)\left|\partial_{n} \psi\right|^{2} \mathrm{~d} \sigma \mathrm{d} t+X_{1}
\end{aligned}
$$

with

$$
\begin{aligned}
X_{1}= & 2 s \lambda \iint_{Q} \varphi a \partial_{x_{i}} \psi \partial_{x_{i}}\left(a \partial_{x_{j}} \beta\right) \partial_{x_{j}} \psi \mathrm{d} x \mathrm{~d} t-s \lambda \iint_{Q} \varphi a \partial_{x_{j}}\left(a \partial_{x_{j}} \beta\right)|\nabla \psi|^{2} \mathrm{~d} x \mathrm{~d} t \\
& -s \lambda \iint_{Q} \varphi\left(\partial_{x_{j}} a\right)\left(a \partial_{x_{j}} \beta\right)|\nabla \psi|^{2} \mathrm{~d} x \mathrm{~d} t .
\end{aligned}
$$

Finally, we get

$$
\begin{aligned}
I_{12^{\prime}}= & -s \lambda^{2} \iint_{Q} \varphi|a \nabla \beta|^{2}|\nabla \psi|^{2} \mathrm{~d} x \mathrm{~d} t+2 s \lambda^{2} \iint_{Q} \varphi(a \nabla \psi \cdot \nabla \beta)^{2} \mathrm{~d} x \mathrm{~d} t \\
& +s \lambda \int_{0}^{T} \int_{S} \varphi\left|a \partial_{n} \psi\right|^{2}\left[\partial_{n} \beta\right]_{S} \mathrm{~d} \sigma \mathrm{d} t-s \lambda \int_{0}^{T} \int_{S} \varphi\left|\nabla_{\tau} \psi\right|^{2}\left(a \partial_{n} \beta\right)[a]_{S} \mathrm{~d} \sigma \mathrm{d} t \\
& -s \lambda \int_{0}^{T} \int_{\Gamma} \varphi\left|a \partial_{n} \psi\right|^{2}\left(\partial_{n} \beta\right) \mathrm{d} \sigma \mathrm{d} t+X_{1},
\end{aligned}
$$

where $X_{1}$ is given by $(63)$, and $[\cdot]_{S}$ denote the jump on $S$. Notice that in (64), due to the choice of $\widetilde{\beta}$ the boundary integrals are nonnegative and this is essential. In fact, from (9) and (10) we have

$$
[a]_{S}=a_{0}-a_{1} \leq 0 \quad \text { on } \quad S
$$

On the other hand, from $(10,31,32)$ and $(43)$, we deduce that

$$
\left[\partial_{n} \beta\right]_{S}=\partial_{n} \beta_{0}-\partial_{n} \beta_{1} \geq 0 \quad \text { on } \quad S
$$

since $n$ is the outward unit normal to $\Omega_{1}$. Moreover, thanks to (29) and (43), we have $\partial_{n} \beta \leq 0$ on $\Gamma$. This justifies the above statement. 
Let us compute the scalar product of the first term of $M_{1} \psi$ and the third one of $M_{2} \psi$.

$$
\begin{aligned}
I_{13^{\prime}}= & -2 s \lambda^{2} \sum_{l=0}^{1} \int_{0}^{T} \int_{\Omega_{l}} \operatorname{div}\left(a_{l} \nabla \psi\right) \varphi a_{l}|\nabla \beta|^{2} \psi \mathrm{d} x \mathrm{~d} t=2 s \lambda^{2} \iint_{Q} a \nabla \psi \nabla\left(\varphi a|\nabla \beta|^{2} \psi\right) \mathrm{d} x \mathrm{~d} t \\
& -2 s \lambda^{2} \int_{0}^{T} \int_{S^{-}} \varphi a_{1}\left(\partial_{n} \psi\right) a_{1}\left|\nabla \beta_{1}\right|^{2} \psi \mathrm{d} \sigma \mathrm{d} t+2 s \lambda^{2} \int_{0}^{T} \int_{S^{+}} \varphi a_{0}\left(\partial_{n} \psi\right) a_{0}\left|\nabla \beta_{0}\right|^{2} \psi \mathrm{d} \sigma \mathrm{d} t \\
= & 2 s \lambda^{2} \iint_{Q} \varphi|a \nabla \beta|^{2}|\nabla \psi|^{2} \mathrm{~d} x \mathrm{~d} t+2 s \lambda^{2} \sum_{l=0}^{1} \int_{0}^{T} \int_{\Omega_{l}} \varphi a_{l} \nabla\left(a_{l}|\nabla \beta|^{2}\right) \nabla \psi \psi \mathrm{d} x \mathrm{~d} t \\
& +2 s \lambda^{3} \iint_{Q} \varphi a^{2} \nabla \beta|\nabla \beta|^{2} \nabla \psi \psi \mathrm{d} x \mathrm{~d} t \\
& -2 s \lambda^{2} \int_{0}^{T} \int_{S^{-}} \varphi a_{1}\left(\partial_{n} \psi\right) a_{1}\left|\partial_{n} \beta_{1}\right|^{2} \psi \mathrm{d} \sigma \mathrm{d} t+2 s \lambda^{2} \int_{0}^{T} \int_{S^{+}} \varphi a_{0}\left(\partial_{n} \psi\right) a_{0}\left|\partial_{n} \beta_{0}\right|^{2} \psi \mathrm{d} \sigma \mathrm{d} t .
\end{aligned}
$$

In (67), again we have used (58) and the fact that $\nabla_{\tau} \beta=0$ on $S$. Finally, we have

$$
I_{13^{\prime}}=2 s \lambda^{2} \iint_{Q} \varphi|a \nabla \beta|^{2}|\nabla \psi|^{2} \mathrm{~d} x \mathrm{~d} t+X_{2}
$$

where

$$
\begin{aligned}
X_{2}= & 2 s \lambda^{2} \iint_{Q} \varphi a \nabla\left(a|\nabla \beta|^{2}\right) \nabla \psi \psi \mathrm{d} x \mathrm{~d} t+2 s \lambda^{3} \iint_{Q} \varphi a^{2} \nabla \beta|\nabla \beta|^{2} \nabla \psi \psi \mathrm{d} x \mathrm{~d} t \\
& +2 s \lambda^{2} \int_{0}^{T} \int_{S} \varphi\left(a \partial_{n} \psi\right)\left(a \partial_{n} \beta\right)\left[\partial_{n} \beta\right]_{S} \psi \mathrm{d} \sigma \mathrm{d} t .
\end{aligned}
$$

The scalar product of the second term of $M_{1} \psi$ with the first one of $M_{2} \psi$ gives

$$
I_{21^{\prime}}=s^{2} \lambda^{2} \iint_{Q} \varphi^{2}|\nabla \beta|^{2} a \partial_{t} \psi \psi \mathrm{d} x \mathrm{~d} t=-s^{2} \lambda^{2} \iint_{Q} \varphi \partial_{t} \varphi|\nabla \beta|^{2} a|\psi|^{2} \mathrm{~d} x \mathrm{~d} t .
$$

We now consider the scalar product between the second term of $M_{1} \psi$ with the second one of $M_{2} \psi$. The following holds:

$$
\begin{aligned}
I_{22^{\prime}}= & -2 s^{3} \lambda^{3} \sum_{l=0}^{1} \int_{0}^{T} \int_{\Omega_{l}} \varphi^{3} a_{l}^{2}|\nabla \beta|^{2}(\nabla \beta) \nabla \psi \psi \mathrm{d} x \mathrm{~d} t \\
= & 3 s^{3} \lambda^{4} \iint_{Q} \varphi^{3} a^{2}|\nabla \beta|^{4}|\psi|^{2} \mathrm{~d} x \mathrm{~d} t+s^{3} \lambda^{3} \sum_{l=0}^{1} \int_{0}^{T} \int_{\Omega_{l}} \varphi^{3} \operatorname{div}\left(a_{l}^{2}|\nabla \beta|^{2} \nabla \beta\right)|\psi|^{2} \mathrm{~d} x \mathrm{~d} t \\
& -s^{3} \lambda^{3} \int_{0}^{T} \int_{S^{-}} \varphi^{3}\left|a_{1} \partial_{n} \beta_{1}\right|^{2}\left(\partial_{n} \beta_{1}\right)|\psi|^{2} \mathrm{~d} \sigma \mathrm{d} t+s^{3} \lambda^{3} \int_{0}^{T} \int_{S^{+}} \varphi^{3}\left|a_{0} \partial_{n} \beta_{0}\right|^{2}\left(\partial_{n} \beta_{0}\right)|\psi|^{2} \mathrm{~d} \sigma \mathrm{d} t \\
= & 3 s^{3} \lambda^{4} \iint_{Q} \varphi^{3} a^{2}|\nabla \beta|^{4}|\psi|^{2} \mathrm{~d} x \mathrm{~d} t+s^{3} \lambda^{3} \int_{0}^{T} \int_{S} \varphi^{3}\left|a \partial_{n} \beta\right|^{2}\left[\partial_{n} \beta\right]_{S}|\psi|^{2} \mathrm{~d} \sigma \mathrm{d} t+X_{3},
\end{aligned}
$$


where

$$
X_{3}=s^{3} \lambda^{3} \sum_{l=0}^{1} \int_{0}^{T} \int_{\Omega_{l}} \varphi^{3} \operatorname{div}\left(a_{l}^{2}|\nabla \beta|^{2} \nabla \beta\right)|\psi|^{2} \mathrm{~d} x \mathrm{~d} t
$$

Observe again that the last boundary integral in (71) is nonnegative because (66) holds.

Now we consider the third and the second terms of $M_{1} \psi$ and $M_{2} \psi$, respectively. Using (45) we can write

$$
\begin{aligned}
I_{32^{\prime}}= & -2 s^{2} \lambda \sum_{l=0}^{1} \int_{0}^{T} \int_{\Omega_{l}} \varphi \partial_{t} \eta a_{l} \nabla \beta \cdot \nabla \psi \psi \mathrm{d} x \mathrm{~d} t=s^{2} \lambda \iint_{Q} \varphi \partial_{t} \eta \operatorname{div}(a \nabla \beta)|\psi|^{2} \mathrm{~d} x \mathrm{~d} t \\
& +s^{2} \lambda^{2} \iint_{Q} \varphi\left(\partial_{t} \eta\right) a|\nabla \beta|^{2}|\psi|^{2} \mathrm{~d} x \mathrm{~d} t-s^{2} \lambda^{2} \iint_{Q} \varphi\left(\partial_{t} \varphi\right) a|\nabla \beta|^{2}|\psi|^{2} \mathrm{~d} x \mathrm{~d} t \\
& -s^{2} \lambda \int_{0}^{T} \int_{S^{-}} \varphi \partial_{t} \eta\left(a_{1} \partial_{n} \beta_{1}\right)|\psi|^{2} \mathrm{~d} \sigma \mathrm{d} t+s^{2} \lambda \int_{0}^{T} \int_{S^{+}} \varphi \partial_{t} \eta\left(a_{0} \partial_{n} \beta_{0}\right)|\psi|^{2} \mathrm{~d} \sigma
\end{aligned}
$$

Since (32) holds we know that $\left.a_{0} \partial_{n} \beta_{0}\right|_{S^{+}}-\left.a_{1} \partial_{n} \beta_{1}\right|_{S^{-}}=0$, then we get

$$
I_{32^{\prime}}=s^{2} \lambda \iint_{Q} \varphi \partial_{t} \eta \operatorname{div}(a \nabla \beta)|\psi|^{2} \mathrm{~d} x \mathrm{~d} t+s^{2} \lambda^{2} \iint_{Q} \varphi\left(\partial_{t} \eta\right) a|\nabla \beta|^{2}|\psi|^{2} \mathrm{~d} x \mathrm{~d} t-s^{2} \lambda^{2} \iint_{Q} \varphi\left(\partial_{t} \varphi\right) a|\nabla \beta|^{2}|\psi|^{2} \mathrm{~d} x \mathrm{~d} t .
$$

The last integrals give

$$
\begin{gathered}
I_{23^{\prime}}=-2 s^{3} \lambda^{4} \iint_{Q} \varphi^{3} a^{2}|\nabla \beta|^{4}|\psi|^{2} \mathrm{~d} x \mathrm{~d} t \\
I_{31^{\prime}}=s \iint_{Q} \partial_{t} \eta \psi \partial_{t} \psi \mathrm{d} x \mathrm{~d} t=-\frac{1}{2} s \iint_{Q} \partial_{t}^{2} \eta|\psi|^{2} \mathrm{~d} x \mathrm{~d} t
\end{gathered}
$$

and

$$
I_{33^{\prime}}=-2 s^{2} \lambda^{2} \iint_{Q} \varphi a \partial_{t} \eta|\nabla \beta|^{2}|\psi|^{2} \mathrm{~d} x \mathrm{~d} t
$$

Finally from (55), taking into account $(56,64,67,70,71,74-76)$ and (77) we deduce that

$$
\begin{aligned}
\left(M_{1} \psi, M_{2} \psi\right)= & s \lambda^{2} \iint_{Q} \varphi|a \nabla \beta|^{2}|\nabla \psi|^{2} \mathrm{~d} x \mathrm{~d} t+2 s \lambda^{2} \iint_{Q} \varphi(a \nabla \psi \cdot \nabla \beta)^{2} \mathrm{~d} x \mathrm{~d} t \\
& +s^{3} \lambda^{4} \iint_{Q} \varphi^{3} a^{2}|\nabla \beta|^{4}|\psi|^{2} \mathrm{~d} x \mathrm{~d} t+s^{3} \lambda^{3} \int_{0}^{T} \int_{S} \varphi^{3}\left|a \partial_{n} \beta\right|^{2}\left[\partial_{n} \beta\right]_{S}|\psi|^{2} \mathrm{~d} \sigma \mathrm{d} t \\
& +s \lambda \int_{0}^{T} \int_{S} \varphi\left|a \partial_{n} \psi\right|^{2}\left[\partial_{n} \beta\right]_{S} \mathrm{~d} \sigma \mathrm{d} t-s \lambda \int_{0}^{T} \int_{S} \varphi\left|\nabla_{\tau} \psi\right|^{2}\left(a \partial_{n} \beta\right)[a]_{S} \mathrm{~d} \sigma \mathrm{d} t \\
& -s \lambda \int_{0}^{T} \int_{\Gamma} \varphi\left|a \partial_{n} \psi\right|^{2}\left(\partial_{n} \beta\right) \mathrm{d} \sigma \mathrm{d} t+X_{1}+X_{2}+I_{21^{\prime}}+X_{3}+I_{31^{\prime}}+I_{32^{\prime}}+I_{33^{\prime}},
\end{aligned}
$$


where $X_{1}, X_{2}, I_{21^{\prime}}, X_{3}, I_{31^{\prime}}, I_{32^{\prime}}$, and $I_{33^{\prime}}$, are given by $(63,69,70,72,74,76)$ and (77) respectively. Thus, we have from (54) the following identity:

$$
\begin{aligned}
\left\|M_{1} \psi\right\|_{2}^{2}+\left\|M_{2} \psi\right\|_{2}^{2} & +2 s \lambda^{2} \iint_{Q} \varphi|a \nabla \beta|^{2}|\nabla \psi|^{2} \mathrm{~d} x \mathrm{~d} t+4 s \lambda^{2} \iint_{Q} \varphi(a \nabla \psi \cdot \nabla \beta)^{2} \mathrm{~d} x \mathrm{~d} t \\
& +2 s^{3} \lambda^{4} \iint_{Q} \varphi^{3} a^{2}|\nabla \beta|^{4}|\psi|^{2} \mathrm{~d} x \mathrm{~d} t+2 s^{3} \lambda^{3} \int_{0}^{T} \int_{S} \varphi^{3}\left|a \partial_{n} \beta\right|^{2}\left[\partial_{n} \beta\right]_{S}|\psi|^{2} \mathrm{~d} \sigma \mathrm{d} t \\
& +2 s \lambda \int_{0}^{T} \int_{S} \varphi\left|a \partial_{n} \psi\right|^{2}\left[\partial_{n} \beta\right]_{S} \mathrm{~d} \sigma \mathrm{d} t-2 s \lambda \int_{0}^{T} \int_{S} \varphi\left|\nabla_{\tau} \psi\right|^{2}\left(a \partial_{n} \beta\right)[a]_{S} \mathrm{~d} \sigma \mathrm{d} t \\
& -2 s \lambda \int_{0}^{T} \int_{\Gamma} \varphi\left|a \partial_{n} \psi\right|^{2}\left(\partial_{n} \beta\right) \mathrm{d} \sigma \mathrm{d} t=\left\|f_{s}\right\|_{2}^{2}-2\left(X_{1}+X_{2}+I_{21^{\prime}}+X_{3}+I_{31^{\prime}}+I_{32^{\prime}}+I_{33^{\prime}}\right) .
\end{aligned}
$$

As we mentioned above, since $\partial_{n} \beta \leq 0$ on $\Gamma$ and thanks to (66) and (65), all the boundary integrals in the left hand side of (79) are nonnegative. Moreover, we know that (9) and (33) hold. Then, for some $\lambda_{0}(\Omega, \omega, a) \geq 1$ we have

$$
\begin{array}{lll}
\lambda^{2} \varphi|a \nabla \beta|^{2} \geq C(\Omega, \omega, a) \lambda^{2} \varphi \quad \text { in } & \left(\Omega \backslash \omega_{0}\right) \times(0, T) & \forall \lambda \geq \lambda_{0}(\Omega, \omega, a), \\
\lambda^{4} \varphi^{3} a^{2}|\nabla \beta|^{4} \geq C(\Omega, \omega, a) \lambda^{4} \varphi^{3} \text { in } \quad & \left(\Omega \backslash \omega_{0}\right) \times(0, T) & \forall \lambda \geq \lambda_{0}(\Omega, \omega, a) .
\end{array}
$$

On the other hand, we use that

$$
\begin{aligned}
\left|a \partial_{x_{j}}\left(a \partial_{x_{j}} \beta\right)\right| & \leq C(\Omega, \omega, a), \\
a|\nabla \beta|^{2} & \leq C(\Omega, \omega, a), \\
\left|\nabla\left(a_{l}|\nabla \beta|^{2}\right)\right| & \leq C(\Omega, \omega, a), \quad l=0,1, \\
\operatorname{div}\left(a_{l}^{2}|\nabla \beta|^{2} \nabla \beta\right) & \leq C(\Omega, \omega, a), \quad l=0,1, \\
\operatorname{div}(a \nabla \beta) & \leq C(\Omega, \omega, a) .
\end{aligned}
$$

Then, it is not difficult to check that for every $\varepsilon>0$, there exists $C_{\varepsilon}>0$ such that

$$
\begin{aligned}
\left|X_{1}\right| \leq & C(\Omega, \omega, a) s \lambda \iint_{Q} \varphi|\nabla \psi|^{2} \mathrm{~d} x \mathrm{~d} t \\
\left|X_{2}\right| \leq & C(\Omega, \omega, a)\left(s \lambda \iint_{Q} \varphi|\nabla \psi|^{2} \mathrm{~d} x \mathrm{~d} t+s \lambda^{3} T^{4} \iint_{Q} \varphi^{3}|\psi|^{2} \mathrm{~d} x \mathrm{~d} t\right) \\
& +\varepsilon s \lambda^{2} \iint_{Q} \varphi|\nabla \psi|^{2} \mathrm{~d} x \mathrm{~d} t+C_{\varepsilon} s \lambda^{4} T^{4} \iint_{Q} \varphi^{3}|\psi|^{2} \mathrm{~d} x \mathrm{~d} t \\
& +\varepsilon s \lambda \int_{0}^{T} \int_{S} \varphi\left|a \partial_{n} \psi\right|^{2}\left[\partial_{n} \beta\right]_{S} \mathrm{~d} \sigma \mathrm{d} t+C_{\varepsilon} s \lambda^{3} T^{4} \int_{0}^{T} \int_{S} \varphi^{3}\left|a \partial_{n} \beta\right|^{2}\left[\partial_{n} \beta\right]_{S}|\psi|^{2} \mathrm{~d} \sigma \mathrm{d} t \\
\left|X_{3}\right| \leq & C(\Omega, \omega, a) s^{3} \lambda^{3} \iint_{Q} \varphi^{3}|\psi|^{2} \mathrm{~d} x \mathrm{~d} t .
\end{aligned}
$$

In the estimate (87), we have used Young inequalities and the fact that

$$
\varphi \leq C \varphi^{3} t^{2}(T-t)^{2} \leq C T^{4} \varphi^{3}
$$


and we will chose $\varepsilon>0$ sufficiently small. Moreover, we have

$$
\begin{aligned}
\left|\partial_{t} \varphi\right| & =\frac{|T-2 t|}{t^{2}(T-t)^{2}} \mathrm{e}^{\lambda \beta} \leq C(\Omega, \omega) T \varphi^{2}, \\
\left|\partial_{t} \eta\right| & =\frac{|T-2 t|\left(\mathrm{e}^{\lambda \bar{\beta}}-\mathrm{e}^{\lambda \beta}\right)}{t^{2}(T-t)^{2}} \leq C(\Omega, \omega) \frac{T \mathrm{e}^{\lambda \bar{\beta}}}{t^{2}(T-t)^{2}} \leq C(\Omega, \omega) \frac{T \mathrm{e}^{2 \lambda \beta}}{t^{2}(T-t)^{2}} \leq C(\Omega, \omega) T \varphi^{2}, \\
\left|\partial_{t t}^{2} \eta\right| & =\frac{2\left|T^{2}-3 T t+3 t^{2}\right|\left(\mathrm{e}^{\lambda \bar{\beta}}-\mathrm{e}^{\lambda \beta}\right)}{t^{3}(T-t)^{3}} \leq \frac{14 T^{2} \mathrm{e}^{\lambda \bar{\beta}}}{t^{3}(T-t)^{3}} \leq C(\Omega, \omega) \frac{T^{2} \mathrm{e}^{2 \lambda \beta}}{t^{3}(T-t)^{3}} \leq C(\Omega, \omega) T^{2} \varphi^{3} .
\end{aligned}
$$

In (90) and (91), we have used that $\mathrm{e}^{\lambda \bar{\beta}} \leq \mathrm{e}^{2 \lambda \beta}$. This is implied by the fact that

$$
\bar{\beta}=\frac{5}{4} \max _{\bar{\Omega}} \beta \leq 2 \min _{\bar{\Omega}} \beta
$$

which is a consequence of the choice of $K$ in (43). Taking into account, equations $(82,85,89,90)$ and (91) we deduce

$$
\begin{aligned}
& \left|I_{21^{\prime}}\right| \leq C(\Omega, \omega, a) T s^{2} \lambda^{2} \iint_{Q} \varphi^{3}|\psi|^{2} \mathrm{~d} x \mathrm{~d} t, \\
& \left|I_{31^{\prime}}\right| \leq C(\Omega, \omega, a) T^{2} s \iint_{Q} \varphi^{3}|\psi|^{2} \mathrm{~d} x \mathrm{~d} t, \\
& \left|I_{32^{\prime}}\right| \leq C(\Omega, \omega, a) T s^{2} \lambda^{2} \iint_{Q} \varphi^{3}|\psi|^{2} \mathrm{~d} x \mathrm{~d} t, \\
& \left|I_{33^{\prime}}\right| \leq C(\Omega, \omega, a) T s^{2} \lambda^{2} \iint_{Q} \varphi^{3}|\psi|^{2} \mathrm{~d} x \mathrm{~d} t .
\end{aligned}
$$

On the other hand, from (53) we can write that

$$
\left\|f_{s}\right\|_{2}^{2} \leq\left\|\mathrm{e}^{-s \eta} f\right\|_{2}^{2}+C(\Omega, \omega, a) s^{2} \lambda^{4} \iint_{Q} \varphi^{2}|\psi|^{2} \leq\left\|\mathrm{e}^{-s \eta} f\right\|_{2}^{2}+C(\Omega, \omega, a) s^{2} \lambda^{4} T^{2} \iint_{Q} \varphi^{3}|\psi|^{2}
$$

Using (80, 86-88, 93-96) and (97) in (79), we obtain:

$$
\begin{aligned}
& \left\|M_{1} \psi\right\|_{2}^{2}+\left\|M_{2} \psi\right\|_{2}^{2}+C s^{3} \lambda^{4} \int_{0}^{T} \int_{\Omega \backslash \omega_{0}} \varphi^{3}|\psi|^{2} \mathrm{~d} x \mathrm{~d} t+C s \lambda^{2} \int_{0}^{T} \int_{\Omega \backslash \omega_{0}} \varphi|\nabla \psi|^{2} \mathrm{~d} x \mathrm{~d} t \\
& +2 s^{3} \lambda^{3} \int_{0}^{T} \int_{S} \varphi^{3}\left|a \partial_{n} \beta\right|^{2}\left[\partial_{n} \beta\right]_{S}|\psi|^{2} \mathrm{~d} \sigma \mathrm{d} t+2 s \lambda \int_{0}^{T} \int_{S} \varphi\left|a \partial_{n} \psi\right|^{2}\left[\partial_{n} \beta\right]_{S} \mathrm{~d} \sigma \mathrm{d} t \\
& \leq\left\|\mathrm{e}^{-s \eta} f\right\|_{2}^{2}+C s \lambda^{3} T^{4} \int_{0}^{T} \int_{S} \varphi^{3}\left|a \partial_{n} \beta\right|^{2}\left[\partial_{n} \beta\right]_{S}|\psi|^{2} \mathrm{~d} \sigma \mathrm{d} t+\varepsilon s \lambda \int_{0}^{T} \int_{S} \varphi\left|a \partial_{n} \psi\right|^{2}\left[\partial_{n} \beta\right]_{S} \mathrm{~d} \sigma \mathrm{d} t \\
& +C s \lambda \iint_{Q} \varphi|\nabla \psi|^{2} \mathrm{~d} x \mathrm{~d} t+\varepsilon s \lambda^{2} \iint_{Q} \varphi|\nabla \psi|^{2} \mathrm{~d} x \mathrm{~d} t+C s^{3} \lambda^{3} \iint_{Q} \varphi^{3}|\psi|^{2} \mathrm{~d} x \mathrm{~d} t \\
& +C s^{2}\left(\lambda^{4} T^{2}+\lambda^{2} T\right) \iint_{Q} \varphi^{3}|\psi|^{2} \mathrm{~d} x \mathrm{~d} t+C s\left(T^{2}+\lambda^{4} T^{4}\right) \iint_{Q} \varphi^{3}|\psi|^{2} \mathrm{~d} x \mathrm{~d} t .
\end{aligned}
$$


From this, for $\lambda \geq \lambda_{1}(\Omega, \omega, a) \geq \lambda_{0}(\Omega, \omega, a)$, with $\lambda_{1}$ not depending on $T$, and for $\varepsilon$ small enough, we can write

$$
\begin{aligned}
\left\|M_{1} \psi\right\|_{2}^{2} & +\left\|M_{2} \psi\right\|_{2}^{2}+s^{3} \lambda^{4} \iint_{Q} \varphi^{3}|\psi|^{2} \mathrm{~d} x \mathrm{~d} t+s \lambda^{2} \iint_{Q} \varphi|\nabla \psi|^{2} \mathrm{~d} x \mathrm{~d} t \\
& +2 s^{3} \lambda^{3} \int_{0}^{T} \int_{S} \varphi^{3}\left|a \partial_{n} \beta\right|^{2}\left[\partial_{n} \beta\right]_{S}|\psi|^{2} \mathrm{~d} \sigma \mathrm{d} t \leq C\left[\left\|\mathrm{e}^{-s \eta} f\right\|_{2}^{2}\right. \\
& +s^{3} \lambda^{4} \int_{0}^{T} \int_{\omega_{0}} \varphi^{3}|\psi|^{2} \mathrm{~d} x \mathrm{~d} t+s \lambda^{2} \int_{0}^{T} \int_{\omega_{0}} \varphi|\nabla \psi|^{2} \mathrm{~d} x \mathrm{~d} t+s\left(T^{2}+\lambda^{4} T^{4}\right) \iint_{Q} \varphi^{3}|\psi|^{2} \mathrm{~d} x \mathrm{~d} t \\
& \left.+s^{2}\left(\lambda^{4} T^{2}+\lambda^{2} T\right) \iint_{Q} \varphi^{3}|\psi|^{2} \mathrm{~d} x \mathrm{~d} t+s \lambda^{3} T^{4} \int_{0}^{T} \int_{S} \varphi^{3}\left|a \partial_{n} \beta\right|^{2}\left[\partial_{n} \beta\right]_{S}|\psi|^{2} \mathrm{~d} \sigma \mathrm{d} t\right]
\end{aligned}
$$

We take now $s \geq \sigma_{0}(\Omega, \omega, a, \lambda)\left(T^{2}+T\right)$, then we also have

$$
\begin{aligned}
\left\|M_{1} \psi\right\|_{2}^{2}+\left\|M_{2} \psi\right\|_{2}^{2}+s^{3} \lambda^{4} \iint_{Q} \varphi^{3}|\psi|^{2} \mathrm{~d} x \mathrm{~d} t+s \lambda^{2} \iint_{Q} \varphi|\nabla \psi|^{2} \mathrm{~d} x \mathrm{~d} t \\
\leq C\left[\left\|\mathrm{e}^{-s \eta} f\right\|_{2}^{2}+s^{3} \lambda^{4} \int_{0}^{T} \int_{\omega_{0}} \varphi^{3}|\psi|^{2} \mathrm{~d} x \mathrm{~d} t+s \lambda^{2} \int_{0}^{T} \int_{\omega_{0}} \varphi|\nabla \psi|^{2} \mathrm{~d} x \mathrm{~d} t\right]
\end{aligned}
$$

Let us deduce from (98) that (46) holds for all $s \geq s_{1}$ where $s_{1}=\sigma_{1}(\Omega, \omega, a, \lambda)\left(T^{2}+T\right)$.

Recall that $\psi=\mathrm{e}^{-s \eta} q$. Then,

$$
\partial_{x_{i}} \psi=\mathrm{e}^{-s \eta}\left(\partial_{x_{i}} q-s \partial_{x_{i}} \eta q\right)=\mathrm{e}^{-s \eta}\left(\partial_{x_{i}} q+s \lambda \varphi \partial_{x_{i}} \beta q\right) .
$$

So we can write that

Consequently, we find the following:

$$
\mathrm{e}^{-s \eta} \partial_{x_{i}} q=\partial_{x_{i}} \psi-s \lambda \mathrm{e}^{-s \eta} \varphi \partial_{x_{i}} \beta q .
$$

$$
\begin{aligned}
s \lambda^{2} \iint_{Q} \mathrm{e}^{-2 s \eta} \varphi|\nabla q|^{2} \mathrm{~d} x \mathrm{~d} t & =s \lambda^{2} \iint_{Q} \varphi\left|\nabla \psi-\mathrm{e}^{-s \eta} s \lambda \varphi \nabla \beta q\right|^{2} \mathrm{~d} x \mathrm{~d} t \\
& \leq C s \lambda^{2} \iint_{Q} \varphi|\nabla \psi|^{2} \mathrm{~d} x \mathrm{~d} t+C(\Omega, \omega) s^{3} \lambda^{4} \iint_{Q} \mathrm{e}^{-2 s \eta} \varphi^{3}|q|^{2} \mathrm{~d} x \mathrm{~d} t .
\end{aligned}
$$

Then, from (98) we have

$$
\begin{aligned}
& s^{3} \lambda^{4} \iint_{Q} \mathrm{e}^{-2 s \eta} \varphi^{3}|q|^{2} \mathrm{~d} x \mathrm{~d} t+s \lambda^{2} \iint_{Q} \mathrm{e}^{-2 s \eta} \varphi|\nabla q|^{2} \mathrm{~d} x \mathrm{~d} t \\
& \leq C(\Omega, \omega, a)\left[\left\|\mathrm{e}^{-s \eta} f\right\|_{2}^{2}+s^{3} \lambda^{4} \int_{0}^{T} \int_{\omega} \mathrm{e}^{-2 s \eta} \varphi^{3}|q|^{2} \mathrm{~d} x \mathrm{~d} t+s \lambda^{2} \int_{0}^{T} \int_{\omega_{0}} \mathrm{e}^{-2 s \eta} \varphi|\nabla q|^{2} \mathrm{~d} x \mathrm{~d} t\right] .
\end{aligned}
$$

In order to conclude the proof of the Carleman inequality (46) it is sufficient to prove that

$$
\begin{aligned}
& s \lambda^{2} \int_{0}^{T} \int_{\omega_{0}} \mathrm{e}^{-2 s \eta} \varphi|\nabla q|^{2} \mathrm{~d} x \mathrm{~d} t \leq C\left[\left\|\mathrm{e}^{-s \eta} f\right\|_{2}^{2}+s^{3} \lambda^{4} \int_{0}^{T} \int_{\omega} \mathrm{e}^{-2 s \eta} \varphi^{3}|q|^{2} \mathrm{~d} x \mathrm{~d} t\right. \\
& \left.+s^{2}\left(\lambda^{4} T^{2}+\lambda^{2} T\right) \int_{0}^{T} \int_{\omega} \mathrm{e}^{-2 s \eta} \varphi^{3}|q|^{2} \mathrm{~d} x \mathrm{~d} t+s\left(\lambda^{3} T^{4}+\lambda^{3} T^{2}+\lambda^{2} T^{3}\right) \int_{0}^{T} \int_{\omega} \mathrm{e}^{-2 s \eta} \varphi^{3}|q|^{2} \mathrm{~d} x \mathrm{~d} t\right]
\end{aligned}
$$


In fact, combining (99) and (100), we deduce that the global Carleman estimate (46) is true for $s \geq \sigma_{1}(\Omega, \omega, a, \lambda)$ $\left(T^{2}+T^{3 / 2}+T\right)$. Notice that it is possible to drop the term in $T^{3 / 2}$ since $T^{3 / 2} \leq 1 / 2\left(T^{2}+T\right)$.

In order to obtain (100), we consider a function $\rho \in C_{0}^{\infty}(\omega)$ such that $\rho \equiv 1$ in $\omega_{0}$ and $\rho \geq 0$. We consider $\omega \subset \Omega_{0}$ and the estimates obtained below remain true for larger $\omega$. Multiplying by $s \lambda \mathrm{e}^{-2 s \eta} \rho \varphi q$ the equation

$$
\partial_{t} q+\operatorname{div}(a \nabla q)=f
$$

and integrating in $\omega \times(0, T)$, we obtain

$$
\frac{s \lambda^{2}}{2} \int_{0}^{T} \int_{\omega} \mathrm{e}^{-2 s \eta} \rho \varphi \partial_{t}|q|^{2}+s \lambda^{2} \int_{0}^{T} \int_{\omega} \mathrm{e}^{-2 s \eta} \rho \varphi \operatorname{div}(a \nabla q) q \mathrm{~d} x \mathrm{~d} t=s \lambda^{2} \int_{0}^{T} \int_{\omega} \mathrm{e}^{-2 s \eta} \rho \varphi f q \mathrm{~d} x \mathrm{~d} t .
$$

In (101), the second term, can be written after integration by parts as follows:

$s \lambda^{2} \int_{0}^{T} \int_{\omega} \mathrm{e}^{-2 s \eta} \rho \varphi \partial_{x_{i}}\left(a \partial_{x_{i}} q\right) q \mathrm{~d} x \mathrm{~d} t=-s \lambda^{2} \int_{0}^{T} \int_{\omega} \mathrm{e}^{-2 s \eta} \rho \varphi a|\nabla q|^{2} \mathrm{~d} x \mathrm{~d} t-\frac{s \lambda^{2}}{2} \int_{0}^{T} \int_{\omega} \partial_{x_{i}}\left(\mathrm{e}^{-2 s \eta} \rho \varphi\right) a \partial_{x_{i}}|q|^{2} \mathrm{~d} x \mathrm{~d} t$.

Then, from (101) we deduce

$$
\begin{aligned}
s \lambda^{2} \int_{0}^{T} \int_{\omega_{0}} \mathrm{e}^{-2 s \eta} \varphi|\nabla q|^{2} \mathrm{~d} x \mathrm{~d} t \leq & \left.C(a) \frac{s \lambda^{2}}{2}\left|\int_{0}^{T} \int_{\omega} \mathrm{e}^{-2 s \eta} \rho \varphi \partial_{t}\right| q\right|^{2} \mathrm{~d} x \mathrm{~d} t\left|+C(a) s \lambda^{2}\right| \int_{0}^{T} \int_{\omega} \mathrm{e}^{-2 s \eta} \rho \varphi f q \mathrm{~d} x \mathrm{~d} t \mid \\
& +\left.C(a) s \lambda^{2}\left|\int_{0}^{T} \int_{\omega} \mathrm{e}^{-2 s \eta} \partial_{x_{i} x_{i}}^{2}\left(\mathrm{e}^{-2 s \eta} \rho \varphi\right)\right| q\right|^{2} \mathrm{~d} x \mathrm{~d} t \mid
\end{aligned}
$$

Let us consider the first term of the right hand side of (102). We have

$$
\begin{aligned}
X_{4}=\frac{s \lambda^{2}}{2} \int_{0}^{T} \int_{\omega} \mathrm{e}^{-2 s \eta} \rho \varphi \partial_{t}|q|^{2} \mathrm{~d} x \mathrm{~d} t & =-\frac{s \lambda^{2}}{2} \int_{0}^{T} \int_{\omega} \partial_{t}\left(\mathrm{e}^{-2 s \eta} \rho \varphi\right)|q|^{2} \mathrm{~d} x \mathrm{~d} t \\
& =s^{2} \lambda^{2} \int_{0}^{T} \int_{\omega} \mathrm{e}^{-2 s \eta} \rho \varphi \partial_{t} \eta|q|^{2} \mathrm{~d} x \mathrm{~d} t-\frac{s \lambda^{2}}{2} \int_{0}^{T} \int_{\omega} \mathrm{e}^{-2 s \eta} \rho \partial_{t} \varphi|q|^{2} \mathrm{~d} x \mathrm{~d} t
\end{aligned}
$$

Using now (89) and (90) in (103) we obtain

$$
\left|X_{4}\right| \leq C s^{2} \lambda^{2} T \int_{0}^{T} \int_{\omega} \mathrm{e}^{-2 s \eta} \rho \varphi^{3}|q|^{2} \mathrm{~d} x \mathrm{~d} t+C s \lambda^{2} T^{3} \int_{0}^{T} \int_{\omega} \mathrm{e}^{-2 s \eta} \rho \varphi^{3}|q|^{2} \mathrm{~d} x \mathrm{~d} t .
$$

On the other hand, for the third term of the right hand side of (102), we can write

$$
\begin{aligned}
\left.s \lambda^{2}\left|\int_{0}^{T} \int_{\omega} \partial_{x_{i} x_{i}}^{2}\left(\mathrm{e}^{-2 s \eta} \rho \varphi\right)\right| q\right|^{2} \mathrm{~d} x \mathrm{~d} t \mid \leq & C s^{3} \lambda^{4} \int_{0}^{T} \int_{\omega} \mathrm{e}^{-2 s \eta} \varphi^{3} \rho|q|^{2} \mathrm{~d} x \mathrm{~d} t \\
& +C\left(s^{2} \lambda^{4} T^{2}+s \lambda^{3} T^{4}+s \lambda^{3} T^{2}\right) \int_{0}^{T} \int_{\omega} \mathrm{e}^{-2 s \eta} \varphi^{3} \rho|q|^{2} \mathrm{~d} x \mathrm{~d} t .
\end{aligned}
$$


This is a consequence of the particular form of $\eta$ and $\varphi$. Indeed, after the following calculation

$$
\begin{gathered}
\partial_{x_{i}}\left(\mathrm{e}^{-2 s \eta} \rho \varphi\right)=\mathrm{e}^{-2 s \eta}\left[\partial_{x_{i}} \rho \varphi+\rho \partial_{x_{i}} \varphi-2 s \rho \varphi \partial_{x_{i}} \eta\right], \\
\partial_{x_{i} x_{j}}^{2}\left(\mathrm{e}^{-2 s \eta} \rho \varphi\right)=\mathrm{e}^{-2 s \eta}\left[-2 s \varphi \partial_{x_{j}} \eta \partial_{x_{i}} \rho-2 s \rho \partial_{x_{j}} \eta \partial_{x_{i}} \varphi+4 s^{2} \varphi \rho \partial_{x_{j}} \eta \partial_{x_{i}} \eta\right. \\
\left.+\varphi \partial_{x_{i} x_{j}}^{2} \rho+\partial_{x_{i}} \rho \partial_{x_{j}} \varphi+\partial_{x_{j}} \rho \partial_{x_{i}} \varphi+\rho \partial_{x_{i} x_{j}}^{2} \varphi-2 s \varphi \partial_{x_{j}} \rho \partial_{x_{i}} \eta-2 s \rho \partial_{x_{j}} \varphi \partial_{x_{i}} \eta-2 s \rho \varphi \partial_{x_{i} x_{j}}^{2} \eta\right]
\end{gathered}
$$

and using the following straightforward estimates

$$
\begin{aligned}
\left|\varphi \partial_{x_{j}} \eta\right| & \leq \lambda \varphi^{2}|\nabla \beta| \leq C \lambda \varphi^{2} \leq C T^{2} \lambda \varphi^{3}, \\
\left|\partial_{x_{j}} \eta \partial_{x_{i}} \varphi\right| & \leq \lambda^{2} \varphi^{2}|\nabla \beta|^{2} \leq C \lambda^{2} \varphi^{2} \leq C T^{2} \lambda^{2} \varphi^{3}, \\
\left|\varphi \partial_{x_{j}} \eta \partial_{x_{i}} \eta\right| & \leq \lambda^{2} \varphi^{3}|\nabla \beta|^{2} \leq C \lambda^{2} \varphi^{3}, \\
\left|\partial_{x_{j}} \varphi\right| & \leq \lambda \varphi|\nabla \beta| \leq C \lambda \varphi \leq C T^{4} \lambda \varphi^{3} \\
\left|\partial_{x_{i} x_{j}}^{2} \varphi\right| & \leq \lambda \varphi|\Delta \beta|+\lambda^{2} \varphi^{2}|\nabla \beta|^{2} \leq C\left(\lambda \varphi+\lambda^{2} \varphi^{2}\right) \leq C\left(T^{4} \lambda+T^{2} \lambda^{2}\right) \varphi^{3} \\
\left|\varphi \partial_{x_{i} x_{j}}^{2} \eta\right| & \leq \lambda \varphi^{2}|\Delta \beta|+\lambda^{2} \varphi^{3}|\nabla \beta|^{2} \leq C T^{2} \lambda \varphi^{3}+C \lambda^{2} \varphi^{3}
\end{aligned}
$$

it is not difficult to see that (105) holds. On the other hand we have

$$
\begin{aligned}
s \lambda^{2}\left|\int_{0}^{T} \int_{\omega} \mathrm{e}^{-2 s \eta} \rho \varphi f q \mathrm{~d} x \mathrm{~d} t\right| & \leq C\left\|\mathrm{e}^{-s \eta} f\right\|_{2}^{2}+C s^{2} \lambda^{4} \int_{0}^{T} \int_{\omega} \mathrm{e}^{-2 s \eta} \varphi^{2} \rho^{2}|q|^{2} \mathrm{~d} x \mathrm{~d} t \\
& \leq C\left\|\mathrm{e}^{-s \eta} f\right\|_{2}^{2}+C s^{2} \lambda^{4} T^{2} \int_{0}^{T} \int_{\omega} \mathrm{e}^{-2 s \eta} \varphi^{3}|q|^{2} \mathrm{~d} x \mathrm{~d} t .
\end{aligned}
$$

Using $(104,105)$ and (106) in (102) we get (100). As we mentioned above, this ends the proof of Carleman inequality (46) of Theorem 3.3 .

The situation of Case 2 is quite different. Let us consider the functions

$$
\beta^{i}=\widetilde{\beta}^{i}+K^{i}, \quad \bar{\beta}^{i}=\frac{5}{4} \max _{\bar{\Omega}} \beta^{i}, \quad \text { for } i=1,2,
$$

with $K^{i}>0$ such that $K^{i} \geq 5 \max _{\bar{\Omega}} \widetilde{\beta}^{i}$, and $\widetilde{\beta}^{i}$ is given by the Lemma 3.2 . We also introduce the following weight functions:

$$
\varphi^{i}(x, t)=\frac{\mathrm{e}^{\lambda \beta^{i}(x)}}{t(T-t)}, \quad \eta^{i}(x, t)=\frac{\mathrm{e}^{\lambda \bar{\beta}^{i}}-\mathrm{e}^{\lambda \beta^{i}}}{t(T-t)}, \quad i=1,2 .
$$

Our second Carleman estimate is the following:

Theorem 3.4. Assume that $\omega \cap \Omega_{0} \neq \emptyset$, a satisfies $(5,6,9)$ and (10) and Condition 2.2 in case (2) is fulfilled. There exists $\lambda_{2}(\Omega, \omega, a)>0$ so that for each $\lambda>\lambda_{2}$ there exists a positive constant $C$ that only depends on $\Omega$, 
$\omega, \mathcal{O}_{1}, \mathcal{O}_{2}$ and $a$, and $s_{6}(\lambda)>0$ so that the following estimate holds

$$
\begin{aligned}
& s^{3} \iint_{Q}\left(\mathrm{e}^{-2 s \eta^{1}}+\mathrm{e}^{-2 s \eta^{2}}\right) t^{-3}(T-t)^{-3}|q|^{2} \mathrm{~d} x \mathrm{~d} t+s \iint_{Q}\left(\mathrm{e}^{-2 s \eta^{1}}+\mathrm{e}^{-2 s \eta^{2}}\right) t^{-1}(T-t)^{-1}|\nabla q|^{2} \mathrm{~d} x \mathrm{~d} t \\
& \leq C s^{3} \iint_{\omega \times(0, T)}\left(\mathrm{e}^{-2 s \eta^{1}}+\mathrm{e}^{-2 s \eta^{2}}\right) t^{-3}(T-t)^{-3}|q|^{2} \mathrm{~d} x \mathrm{~d} t \\
& \quad+C \iint_{Q}\left(\mathrm{e}^{-2 s \eta^{1}}+\mathrm{e}^{-2 s \eta^{2}}\right)\left|\partial_{t} q+\operatorname{div}(a(x) \nabla q)\right|^{2} \mathrm{~d} x \mathrm{~d} t
\end{aligned}
$$

for all $q \in Z_{0}$ and $s \geq s_{6}$. Moreover, $s_{6}$ is of the form $s_{6}=\sigma_{6}\left(\Omega, \omega, \mathcal{O}_{1}, \mathcal{O}_{2}, a, \lambda\right)\left(T^{2}+T\right)$, where $\sigma_{6}$ is a positive constant that only depends on $\Omega, \omega, \mathcal{O}_{1}, \mathcal{O}_{2}$, a and $\lambda$.

Proof of Theorem 3.4. In order to obtain (109), we will apply the global Carleman inequality (46) from Theorem 3.3 and the properties of weight functions (108).

We observe, that from Lemma 3.2, we know that $\nabla \beta^{i}$ can vanish only in $\omega_{0}$ and $B_{i}$ for $i=1,2$, where the open subsets $B_{1}$ and $B_{2}$ are fixed balls defined in Lemma 3.2. Taking into account these statements, we can use two weight functions given by (108) and write two Carleman estimates like (46). More precisely, there exist a positive constant $C$ and $s_{1}$ that only depends on $\Omega \omega$ and $a$, such that

$$
\begin{aligned}
& s^{3} \iint_{Q} \mathrm{e}^{-2 s \eta^{i}} t^{-3}(T-t)^{-3}|q|^{2} \mathrm{~d} x \mathrm{~d} t+s \iint_{Q} \mathrm{e}^{-2 s \eta^{i}} t^{-1}(T-t)^{-1}|\nabla q|^{2} \mathrm{~d} x \mathrm{~d} t \\
& \leq C\left(s^{3} \iint_{\omega \times(0, T)} \mathrm{e}^{-2 s \eta^{i}} t^{-3}(T-t)^{-3}|q|^{2} \mathrm{~d} x \mathrm{~d} t+s^{3} \iint_{\widetilde{B}_{i} \times(0, T)} \mathrm{e}^{-2 s \eta^{i}} t^{-3}(T-t)^{-3}|q|^{2} \mathrm{~d} x \mathrm{~d} t\right. \\
& \left.\quad+\iint_{Q} \mathrm{e}^{-2 s \eta^{i}}\left|\partial_{t} q+\operatorname{div}(a(x) \nabla q)\right|^{2} \mathrm{~d} x \mathrm{~d} t\right)
\end{aligned}
$$

for $i=1,2$, for all $q \in Z_{0}$ and $s \geq s_{1}$. Moreover, $s_{1}$ is of the form $s_{1}=\sigma_{1}(\Omega, \omega, a, \lambda)\left(T^{2}+T\right)$.

Let us show that from (110), using the properties of the functions $\beta^{1}$ and $\beta^{2}$, we can deduce the Carleman estimate (109). For this, it will be sufficient to see that for each $C>0$, there exists $s_{4}$ such that

$$
\begin{array}{ll}
\mathrm{e}^{-2 s \eta^{2}} \geq 2 C \mathrm{e}^{-2 s \eta^{1}} & \text { in } \widetilde{B}_{1}, \\
\mathrm{e}^{-2 s \eta^{1}} \geq 2 C \mathrm{e}^{-2 s \eta^{2}} & \text { in } \widetilde{B}_{2}
\end{array}
$$

for $s \geq s_{4}=\sigma_{4}\left(\Omega, \omega, \mathcal{O}_{1}, \mathcal{O}_{2}, \lambda\right) T^{2}$.

Indeed, adding the two Carleman inequality (110), we deduce

$$
\begin{aligned}
& s^{3} \iint_{Q}\left(\mathrm{e}^{-2 s \eta^{1}}+\mathrm{e}^{-2 s \eta^{2}}\right) t^{-3}(T-t)^{-3}|q|^{2} \mathrm{~d} x \mathrm{~d} t+s \iint_{Q}\left(\mathrm{e}^{-2 s \eta^{1}}+\mathrm{e}^{-2 s \eta^{2}}\right) t^{-1}(T-t)^{-1}|\nabla q|^{2} \mathrm{~d} x \mathrm{~d} t \\
& \leq C s^{3} \iint_{\omega \times(0, T)}\left(\mathrm{e}^{-2 s \eta^{1}}+\mathrm{e}^{-2 s \eta^{2}}\right) t^{-3}(T-t)^{-3}|q|^{2} \mathrm{~d} x \mathrm{~d} t \\
& \quad+C\left(s^{3} \iint_{\widetilde{B}_{1} \times(0, T)} \mathrm{e}^{-2 s \eta^{1}} t^{-3}(T-t)^{-3}|q|^{2} \mathrm{~d} x \mathrm{~d} t+s^{3} \iint_{\widetilde{B}_{2} \times(0, T)} \mathrm{e}^{-2 s \eta^{2}} t^{-3}(T-t)^{-3}|q|^{2} \mathrm{~d} x \mathrm{~d} t\right) \\
& \quad+C \iint_{Q}\left(\mathrm{e}^{-2 s \eta^{1}}+\mathrm{e}^{-2 s \eta^{2}}\right)\left|\partial_{t} q+\operatorname{div}(a(x) \nabla q)\right|^{2} \mathrm{~d} x \mathrm{~d} t
\end{aligned}
$$

for $s \geq s_{5}=\max \left(s_{1}, s_{4}\right)$. 
On the other hand, according to (111) and (112), we obtain that

$$
\begin{aligned}
& C s^{3} \iint_{\widetilde{B}_{1} \times(0, T)} \mathrm{e}^{-2 s \eta^{1}} t^{-3}(T-t)^{-3}|q|^{2} \mathrm{~d} x \mathrm{~d} t+C s^{3} \iint_{\widetilde{B}_{2} \times(0, T)} \mathrm{e}^{-2 s \eta^{2}} t^{-3}(T-t)^{-3}|q|^{2} \mathrm{~d} x \mathrm{~d} t \\
& \leq \frac{s^{3}}{2} \iint_{\widetilde{B}_{1} \times(0, T)} \mathrm{e}^{-2 s \eta^{2}} t^{-3}(T-t)^{-3}|q|^{2} \mathrm{~d} x \mathrm{~d} t+\frac{s^{3}}{2} \iint_{\widetilde{B}_{2} \times(0, T)} \mathrm{e}^{-2 s \eta^{1}} t^{-3}(T-t)^{-3}|q|^{2} \mathrm{~d} x \mathrm{~d} t \\
& \leq \frac{s^{3}}{2} \iint_{Q}\left(\mathrm{e}^{-2 s \eta^{1}}+\mathrm{e}^{-2 s \eta^{2}}\right) t^{-3}(T-t)^{-3}|q|^{2} \mathrm{~d} x \mathrm{~d} t
\end{aligned}
$$

for $s \geq s_{6}=\sigma_{6}\left(\Omega, \omega, \mathcal{O}_{1}, \mathcal{O}_{2}, \lambda\right) T^{2}$. Combining (113) and (114), we find (109).

To conclude the proof, let us justify (111) and (112). By construction, we have that

$$
\beta_{1}^{2} \geq 2 \beta_{1}^{1} \quad \text { in } \widetilde{B}_{1}
$$

Using (115), we can deduce that for all $\lambda \geq 1$ there exists a positive constant $\alpha$, which only depends on $\Omega$, $\omega$, $\mathcal{O}_{1}, \mathcal{O}_{2}$ such that

$$
\eta^{1}-\eta^{2} \geq \alpha \eta^{1} \quad \text { in } \widetilde{B}_{1}
$$

Indeed, equation (116) is a consequence of the following:

$$
\eta^{1}-\eta^{2}=\frac{\mathrm{e}^{\lambda \beta_{1}^{2}}-\mathrm{e}^{\lambda \beta_{1}^{1}}}{t(T-t)} \geq \frac{\mathrm{e}^{2 \lambda \beta_{1}^{1}}-\mathrm{e}^{\lambda \beta_{1}^{1}}}{t(T-t)} \geq \alpha \frac{\mathrm{e}^{\lambda \bar{\beta}^{1}}-\mathrm{e}^{\lambda \beta_{1}^{1}}}{t(T-t)}=\alpha \eta^{1} \quad \text { in } \widetilde{B}_{1} .
$$

Then, from (116) we obtain that, for each $C>0$, there exists $s_{2}$ such that

$$
\frac{\mathrm{e}^{-2 s \eta^{2}}}{\mathrm{e}^{-2 s \eta^{1}}} \geq \mathrm{e}^{2 s \alpha \eta^{1}} \geq \mathrm{e}^{2 s \alpha \min \eta^{1}} \geq 2 C \text { in } \widetilde{B}_{1}
$$

for $s \geq s_{2}=\sigma_{2}\left(\Omega, \omega, \mathcal{O}_{1}, \mathcal{O}_{2}\right) T^{2}$. This is exactly the inequality (111) for $s \geq s_{2}$.

By similar arguments, using the fact that

$$
\beta_{1}^{1} \geq 2 \beta_{1}^{2} \text { in } \mathcal{O}_{2}
$$

it is easy to see that, for each $C>0$, there exists $s_{3}$ such that

$$
\frac{\mathrm{e}^{-2 s \eta^{1}}}{\mathrm{e}^{-2 s \eta^{2}}} \geq 2 C \text { in } \widetilde{B}_{2}
$$

for $s \geq s_{3}=\sigma_{3}\left(\Omega, \omega, \mathcal{O}_{1}, \mathcal{O}_{2}\right) T^{2}$. Then, equation (112) holds for $s \geq s_{3}$. Taking now $s_{4}=\max \left(s_{2}, s_{3}\right)$ we get (111) and (112) for $s \geq s_{4}$. This ends the proof of Theorem 3.4.

\section{ObSeRvability Inequalities AND teChNiCAL Results}

In this section we will deduce some observability estimates as a consequence of appropriate global Carleman inequalities and regularizing effect of the heat equation. This will be needed to prove the null controllability result for a linear transmission problem with controls in $L^{r}\left(0, T ; L^{r}(\omega)\right)$ with $r$ sufficiently large, such that (4) holds. 
Let us consider the following linear (adjoint) transmission problem:

$$
\left\{\begin{array}{cc}
-\partial_{t} q-\operatorname{div}(a(x) \nabla q)+b q=0 & \text { in } Q, \\
q=0 & \text { on } \Sigma, \\
q(x, T)=q_{T} & \text { in } \Omega,
\end{array}\right.
$$

where $a$ satisfies $(5,6,9)$ and $(10), b \in L^{\infty}(Q)$, and $q_{T} \in L^{2}(\Omega)$. First of all, let us prove the observability estimate with $L^{2}\left(0, T ; L^{2}(\omega)\right)$-norm in the right hand side. This can be used to deduce null controllability result (and estimates) for linear transmition problem with bounded potential, with controls in $L^{2}\left(0, T ; L^{2}(\omega)\right.$ ). We have the following:

Proposition 4.1. Assume that $\omega \cap \Omega_{0} \neq \emptyset$ and that Condition 2.1 (resp. Condition 2.2) in case (1) (resp. in case (2)) are fulfilled. Then for any a satisfying $(5,6,9,10), b \in L^{\infty}(Q)$ and $q_{T} \in L^{2}(\Omega)$, there exists a positive constant $C$ that only depends on $\Omega, \omega$ and a (resp. $\Omega, \omega \mathcal{O}_{1}, \mathcal{O}_{2}$ and a), such that

$$
\|q(\cdot, 0)\|_{L^{2}(\Omega)}^{2} \leq \exp \left[C\left(1+\frac{1}{T}+T\|b\|_{\infty}+\|b\|_{\infty}^{2 / 3}\right)\right] \iint_{\omega \times(0, T)}|q|^{2} \mathrm{~d} x \mathrm{~d} t
$$

where $q$ is the solution to the corresponding system (118).

For simplicity, we only present the proof of Proposition 4.1 for the situation (1). We just note, that the proof corresponding to the situation (2) is similar, it suffices to take into account the different estimates (in space) for the weight functions that appear in the global Carleman inequality (109). Thus we also obtain the constants depending on $\mathcal{O}_{1}$ and $\mathcal{O}_{2}$.

Proof of Proposition 4.1. We will use global Carleman inequality (46) from Theorem 3.3 and some estimates for the weight functions. Let $b$ and $q_{T}$ be given and let $q$ be the solution to (118).

Step 1: We will first see that

$$
\iint_{\Omega \times(T / 4,3 T / 4)}|q|^{2} \mathrm{~d} x \mathrm{~d} t \leq \exp \left[C\left(1+\frac{1}{T}+\|b\|_{\infty}^{2 / 3}\right)\right] \iint_{\omega \times(0, T)}|q|^{2} \mathrm{~d} x \mathrm{~d} t .
$$

By density, we can write (46) for $q$ being the solution of (118). This gives

$$
\begin{aligned}
s^{3} \iint_{Q} \mathrm{e}^{-2 s \eta} t^{-3}(T-t)^{-3}|q|^{2} \mathrm{~d} x \mathrm{~d} t \leq & C\left(s^{3} \iint_{\omega \times(0, T)} \mathrm{e}^{-2 s \eta} t^{-3}(T-t)^{-3}|q|^{2} \mathrm{~d} x \mathrm{~d} t\right. \\
& \left.+\iint_{Q} \mathrm{e}^{-2 s \eta}|b q|^{2} \mathrm{~d} x \mathrm{~d} t\right)
\end{aligned}
$$

for all $s \geq s_{1}$. We can estimate the second term in the right as follows:

$$
\iint_{Q} \mathrm{e}^{-2 s \eta}|b q|^{2} \mathrm{~d} x \mathrm{~d} t \leq 2^{-6} T^{6}\|b\|_{\infty}^{2} \iint_{Q} \mathrm{e}^{-2 s \eta} t^{-3}(T-t)^{-3}|q|^{2} \mathrm{~d} x \mathrm{~d} t .
$$

Thus, we deduce from (121) that

$$
\iint_{Q} \mathrm{e}^{-2 s \eta} t^{-3}(T-t)^{-3}|q|^{2} \mathrm{~d} x \mathrm{~d} t \leq C \iint_{\omega \times(0, T)} \mathrm{e}^{-2 s \eta} t^{-3}(T-t)^{-3}|q|^{2} \mathrm{~d} x \mathrm{~d} t
$$


provided

$$
s \geq s_{7}=\max \left(s_{1}, C T^{2}\|b\|_{\infty}^{2 / 3}\right)
$$

On the other hand, it can be easily verified that

$$
\mathrm{e}^{-2 s \eta} t^{-3}(t-T)^{-3} \leq 2^{6} T^{-6} \exp \left(-C s T^{-2}\right) \quad \forall(x, t) \in \bar{Q}
$$

and

$$
\mathrm{e}^{-2 s \eta} t^{-3}(t-T)^{-3} \geq\left(\frac{16}{3}\right)^{3} T^{-6} \exp \left(-C s T^{-2}\right) \quad \forall(x, t) \in \bar{\Omega} \times[T / 4,3 T / 4]
$$

whenever

$$
s \geq s_{8}=\max \left(s_{7}, C T^{2}\right)
$$

(constants $C$ in (124) and (125) may be different). If we analyze the structure of the constants $s_{7}$ and $s_{8}$, we see that $s_{8} \leq s_{9}$, where $s_{9}$ is of the form

$$
s_{9}=\sigma_{9}(\Omega, \omega, a)\left(T+T^{2}+T^{2}\|b\|_{\infty}^{2 / 3}\right) .
$$

Let us fix the constant $s=s_{9}$. We write (122) for $s=s_{9}$ taking into account (124) and (125) and we deduce that (120) is satisfied for any solution $q$ of (118).

Step 2: Let us now prove that

$$
\|q(x, 0)\|_{2}^{2} \leq \exp \left[C\left(\frac{1}{T}+T\|b\|_{\infty}\right)\right] \iint_{\Omega \times(T / 4,3 T / 4)}|q|^{2} \mathrm{~d} x \mathrm{~d} t .
$$

The estimate (127) together with (120) leads to the desired observability inequality (119).

Let $\theta_{0} \in C^{1}[0,1]$ be a function such that, $0 \leq \theta_{0} \leq 1, \theta_{0}=1$ in $[0,1 / 4], \theta_{0}=0$ in $[3 / 4,1]$. Now, we consider a function $\theta(t)=\theta_{0}(t / T)$ and we write (118) for $\theta(t) q$. We obtain

$$
\begin{cases}-\partial_{t}(\theta q)-\operatorname{div}(a(x) \nabla(\theta q))+b(\theta q)=-q \partial_{t} \theta & \text { in } \Omega \times(0,3 T / 4), \\ \theta q=0 & \text { on } \partial \Omega \times(0,3 T / 4), \\ \theta q(x, 3 T / 4)=0 & \text { in } \Omega .\end{cases}
$$

Multiplying (128) by $\theta q$ and integrating in $\Omega$, we have

$$
-\frac{1}{2} \frac{\mathrm{d}}{\mathrm{d} t} \int_{\Omega}|\theta q|^{2} \mathrm{~d} x+\int_{\Omega} a|\nabla(\theta q)|^{2} \mathrm{~d} x=-\int_{\Omega} b|\theta q|^{2} \mathrm{~d} x-\int_{\Omega} \theta\left(\partial_{t} \theta\right)|q|^{2} \mathrm{~d} x \quad \forall t \geq 0 .
$$

Thus,

and

$$
-\frac{\mathrm{d}}{\mathrm{d} t} \int_{\Omega}|\theta q|^{2} \mathrm{~d} x+2 \int_{\Omega} a|\nabla(\theta q)|^{2} \mathrm{~d} x \leq\left. 2|| b\left|\|_{\infty} \int_{\Omega}\right| \theta q\right|^{2} \mathrm{~d} x+2 \int_{\Omega} \theta\left|\partial_{t} \theta\right||q|^{2} \mathrm{~d} x
$$

$$
-\frac{\mathrm{d}}{\mathrm{d} t}\left(\exp \left(2\|b\|_{\infty} t\right) \int_{\Omega}|\theta q|^{2} \mathrm{~d} x\right) \leq 2 \exp \left(2\|b\|_{\infty} t\right) \int_{\Omega} \theta\left|\partial_{t} \theta \| q\right|^{2} \mathrm{~d} x
$$


for all $t \geq 0$. Integrating this inequality with respect to time in $[0, t]$ with $t \in[3 T / 4, T]$, we obtain

$$
\begin{aligned}
\int_{\Omega}|q(x, 0)|^{2} \mathrm{~d} x & \leq \int_{0}^{t} \exp \left(2\|b\|_{\infty} t\right) 2 \int_{\Omega}|q|^{2} \theta \partial_{t} \theta \mathrm{d} x \\
& \leq \frac{2 C}{T} \exp \left(\frac{3 T}{2}\|b\|_{\infty}\right) \iint_{\Omega \times(T / 4,3 T / 4)}|q|^{2} \mathrm{~d} x \mathrm{~d} t .
\end{aligned}
$$

In (130), we have used the fact that $\theta \leq 1$ and $\left|\partial_{t} \theta\right|=\left|\partial_{t} \theta_{0}(t / T)\right| / T \leq C / T$. This justifies the estimate (127) and ends the proof of Proposition 4.1.

As we mentioned above, to analyze the controllability for nonlinear problem (8) we need the controls in $L^{r}\left(0, T ; L^{r}(\omega)\right)$ for $r$ sufficiently large $(1 / r+N /(2 r)<1)$. For this we are going to prove a refined version of the observability inequality (119), i.e. with $L^{r^{\prime}}\left(0, T ; L^{r^{\prime}}(\omega)\right)$-norm in the right hand side, where $r^{\prime}$ is the dual exponent to $r$. We have:

Proposition 4.2. Assume that $\omega \cap \Omega_{0} \neq \emptyset$ and that Condition 2.1 (resp. Condition 2.2) in case (1) (resp. in case (2)) are fulfilled. Then for any a satisfying $(5,6,9)$ and $(10), b \in L^{\infty}(Q), q_{T} \in L^{2}(\Omega)$ and any $r^{\prime}$ sufficiently small, there exist a positive constant $C$ that only depends on $\Omega, \omega, a, r^{\prime}$ and $N$ (resp. $\Omega, \omega \mathcal{O}_{1}, \mathcal{O}_{2}$, $a, r^{\prime}$ and $N$ ) and a positive constant $\widetilde{C}$ depending on $\Omega, \omega$, a (resp. $\Omega, \omega \mathcal{O}_{1}, \mathcal{O}_{2}$ and a) such that

$$
\|q(\cdot, 0)\|_{L^{2}(\Omega)}^{2}+\iint_{Q} \mathrm{e}^{-2 s \widetilde{C} T^{-1} /(T-t)}(T-t)^{-3}|q|^{2} \mathrm{~d} x \mathrm{~d} t \leq \exp \left[C H\left(T,\|b\|_{\infty}\right)\right]\left(\iint_{\omega \times(0, T)}|q|^{r^{\prime}} \mathrm{d} x \mathrm{~d} t\right)^{2 / r^{\prime}}
$$

for all $s \geq \sigma(\Omega, \omega, a)\left(T^{2}+T+T^{2}\|b\|_{\infty}^{2 / 3}\right)$, where $\sigma$ is a positive constant depending on $\Omega, \omega$ and a (resp. $\Omega, \omega$, $\left.a, \mathcal{O}_{1}, \mathcal{O}_{2}\right), H\left(T,\|b\|_{\infty}\right)$ is given by

$$
H\left(T,\|b\|_{\infty}\right)=1+\frac{1}{T}+T+\left(T+T^{1 / 2}\right)\|b\|_{\infty}+\|b\|_{\infty}^{2 / 3}
$$

and $q$ is the solution to the corresponding system (118).

As before, for simplicity, we only present the proof corresponding to case (1). We take into account the estimates for the weight functions in (109) for treatment of case (2). In the sequel, $\sigma(\Omega, \omega, a)$ will stand for a generic positive constant only depending on $\Omega, \omega$ and $a$, whose value can change from line to line. Let us first prove the following technical lemma:

Lemma 4.3. Let $\widetilde{\omega}$ be a nonempty open set such that $\widetilde{\omega} \subset \subset \omega$. Then, for any a satisfying (5, 6) and (9), $b \in L^{\infty}(Q), q_{T} \in L^{2}(\Omega)$ and any $r^{\prime}$ sufficiently small, there exists $C=C(\Omega, \omega, a)>0$ such that

$$
\iint_{\widetilde{\omega} \times(0, T)} \mathrm{e}^{-2 s \eta} t^{-3}(T-t)^{-3}|q|^{2} \mathrm{~d} x \mathrm{~d} t \leq C T^{-3} T^{\alpha} K\left(T,\|b\|_{\infty}\right)^{\gamma} \mathrm{e}^{-C s T^{-2}}\left(\iint_{\omega \times(0, T)}|q|^{r^{\prime}} \mathrm{d} x \mathrm{~d} t\right)^{2 / r^{\prime}}
$$

for all $s \geq \sigma(\Omega, \omega, a) T^{2}$, where $\sigma$ is a positive constant depending on $\Omega, \omega$ and $a, \alpha, \gamma$ are positive numbers only depending of $N, K\left(T,\|b\|_{\infty}\right)$ is given by

$$
K\left(T,\|b\|_{\infty}\right)=1+T^{1 / 2}\left(1+\|b\|_{\infty}\right)+T^{-5 / 2}\left(s+T^{2}\right)
$$

and $q$ is the solution to the corresponding system (118). 
Proof of Lemma 4.3. Let $\widetilde{\omega}$ be a nonempty open set such that $\widetilde{\omega} \subset \subset \omega$. Notice that without loss of generality we can consider $\omega \subset \subset \Omega_{0}$ or $\omega \subset \subset \Omega_{1}$ with a smooth boundary and the estimates obtained below remain true for a larger $\omega$. Let us consider a function $\theta \in \mathcal{D}(\omega)$, such that $\theta=1$ in $\widetilde{\omega}$. We set

$$
w(x, t)=\theta(x) \varphi(x, t) q(x, t)
$$

where $q$ is the solution of (118) and $\varphi$ is given by

$$
\varphi(x, t)=\frac{\mathrm{e}^{-s \eta}}{t^{3 / 2}(T-t)^{3 / 2}} .
$$

Notice that $w(T)=w(0)=0$. Taking into account (118), we deduce that $w$ satisfy the following problem:

$$
\begin{cases}-\partial_{t} w-\operatorname{div}(a(x) \nabla w)=-b \theta q \varphi-\theta q \partial_{t} \varphi-2 a \nabla(\theta \varphi) \cdot \nabla q-\operatorname{div}(a \nabla(\theta \varphi)) q & \text { in } Q \\ w=0 & \text { on } \Sigma \\ w(x, T)=0 & \text { in } \Omega .\end{cases}
$$

For simplicity of the computations, we put $\widetilde{w}(x, t)=\widetilde{w}(x, T-t)$ for $(x, t) \in Q$. In a similar way, we introduce the functions $\widetilde{a}, \widetilde{b}, \widetilde{\varphi}$ and $\widetilde{q}$. Then we have

$$
\begin{cases}\partial_{t} \widetilde{w}-\operatorname{div}(\widetilde{a}(x) \nabla \widetilde{w})=-\widetilde{b} \theta \widetilde{q} \widetilde{\varphi}+\theta \widetilde{q} \partial_{t} \widetilde{\varphi}-2 \widetilde{a} \nabla(\theta \widetilde{\varphi}) \cdot \nabla \widetilde{q}-\operatorname{div}(\widetilde{a} \nabla(\theta \widetilde{\varphi})) \widetilde{q} & \text { in } Q \\ \widetilde{w}=0 & \text { on } \Sigma \\ \widetilde{w}(x, 0)=0 & \text { in } \Omega\end{cases}
$$

On the other hand, let $z$ be the solution of the problem

$$
\begin{cases}-\partial_{\tau} z-\operatorname{div}(a(x) \nabla z)=0 & \text { in } \omega \times(0, t) \\ z=0 & \text { on } \partial \omega \times(0, t) \\ z(x, t)=\psi & \text { in } \omega\end{cases}
$$

where $\psi \in L^{2}(\Omega)$ is given. Multiplying (137) by $z$ and integrating in $\omega$ and in $\tau \in(0, t)$, we obtain the following for $t \in(0, T)$ :

$$
\begin{aligned}
(\widetilde{w}(t), z(t))= & \int_{0}^{t} \int_{\omega}\left(-\widetilde{b} \theta \widetilde{\varphi}+\theta \partial_{t} \widetilde{\varphi}-\operatorname{div}(\widetilde{a} \nabla(\theta \widetilde{\varphi}))\right) \widetilde{q} z \mathrm{~d} x \mathrm{~d} \tau-2 \int_{0}^{t} \int_{\omega} \widetilde{a} \nabla(\theta \widetilde{\varphi}) \cdot \nabla \widetilde{q} z \mathrm{~d} x \mathrm{~d} \tau \\
\leq & C\left(1+\|b\|_{\infty}\right) \int_{0}^{t}|\widetilde{\varphi}|\|\widetilde{q}\|_{L^{r^{\prime}(\omega)}}\|z\|_{L^{r}(\omega)} \mathrm{d} \tau+C \int_{0}^{t}|\widetilde{\varphi}|\|\widetilde{q}\|_{L^{r^{\prime}(\omega)}}\|\nabla z\|_{L^{r}(\omega)} \mathrm{d} \tau \\
& +\int_{0}^{t}\left|\partial_{t} \widetilde{\varphi}\right|\|\widetilde{q}\|_{L^{r^{\prime}(\omega)}}\|z\|_{L^{r}(\omega)} \mathrm{d} \tau
\end{aligned}
$$

where $C$ is a positive constant depending on $\omega, \widetilde{\omega}($ i.e. on $\omega)$ and $a$. In (139), we have used that $|\nabla \widetilde{\varphi}| \leq C|\widetilde{\varphi}|$ and that $|\Delta \widetilde{\varphi}| \leq C|\widetilde{\varphi}|$.

Notice that, since the diffusion coefficients are sufficiently regular in $\omega$ and thanks to the regularizing effect of the heat equation ( $c f$. [19] and [6]), we know that for all $t>0$ and $1 \leq p, q \leq+\infty$ the following holds:

$$
\begin{array}{r}
\|S(t) u\|_{L^{p}(\omega)} \leq C t^{-\frac{N}{2}\left(\frac{1}{q}-\frac{1}{p}\right)}\|u\|_{L^{q}(\omega)} \forall u \in L^{q}(\omega), \\
\|S(t) u\|_{W^{1, p}(\omega)} \leq C t^{-\frac{N}{2}\left(\frac{1}{q}-\frac{1}{p}\right)-\frac{1}{2}}\|u\|_{L^{q}(\omega)} \forall u \in L^{q}(\omega),
\end{array}
$$

where $\{S(t): t \geq 0\}$ denotes the semigroup generated by the heat equation with Dirichlet boundary conditions. 
We apply to $z$ (solution of (138)) the estimates (140) and (141) with $q=2$ and $p=r$, so we can write from (139) that

$$
\begin{aligned}
(\widetilde{w}(t), z(t)) \leq & C\left(1+\|b\|_{\infty}\right) \int_{0}^{t}|\widetilde{\varphi}|(t-\tau)^{-\frac{N}{2}\left(\frac{1}{2}-\frac{1}{r}\right)}\|\widetilde{q}(\cdot, \tau)\|_{L^{r^{\prime}}(\omega)}\|\psi\|_{L^{2}(\omega)} \mathrm{d} \tau \\
& +C \int_{0}^{t}|\widetilde{\varphi}|(t-\tau)^{-\frac{N}{2}\left(\frac{1}{2}-\frac{1}{r}\right)-\frac{1}{2}}\|\widetilde{q}(\cdot, \tau)\|_{L^{r^{\prime}(\omega)}}\|\psi\|_{L^{2}(\omega)} \mathrm{d} \tau \\
& +C \int_{0}^{t}\left|\partial_{t} \widetilde{\varphi}\right|(t-\tau)^{-\frac{N}{2}\left(\frac{1}{2}-\frac{1}{r}\right)}\|\widetilde{q}(\cdot, \tau)\|_{L^{r^{\prime}(\omega)}}\|\psi\|_{L^{2}(\omega)} \mathrm{d} \tau
\end{aligned}
$$

for all $t \in[0, T]$. Thus, from (142) we have

$$
\begin{aligned}
\|\widetilde{w}(\cdot, t)\|_{L^{2}(\widetilde{\omega})}= & \sup _{\|\psi\|_{L^{2}(\omega)}=1}(\widetilde{w}(\cdot, t), \psi) \leq C\left(1+\|b\|_{\infty}\right) \int_{0}^{t}|\widetilde{\varphi}|(t-\tau)^{-\frac{N}{2}\left(\frac{1}{2}-\frac{1}{r}\right)}\|\widetilde{q}(\cdot, \tau)\|_{L^{r^{\prime}}(\omega)} \mathrm{d} \tau \\
& +C \int_{0}^{t}|\widetilde{\varphi}|(t-\tau)^{-\frac{N}{2}\left(\frac{1}{2}-\frac{1}{r}\right)-\frac{1}{2}}\|\widetilde{q}(\cdot, \tau)\|_{L^{r^{\prime}}(\omega)} \mathrm{d} \tau+C \int_{0}^{t}\left|\partial_{t} \widetilde{\varphi}\right|(t-\tau)^{-\frac{N}{2}\left(\frac{1}{2}-\frac{1}{r}\right)}\|\widetilde{q}(\cdot, \tau)\|_{L^{r^{\prime}(\omega)}} \mathrm{d} \tau
\end{aligned}
$$

for all $t \in[0, T]$. This gives,

$$
\begin{aligned}
\|\widetilde{w}(\cdot, t)\|_{L^{2}(\widetilde{\omega}) \leq} & C\left(1+T^{1 / 2}+T^{1 / 2}\|b\|_{\infty}\right) \int_{0}^{t}|\widetilde{\varphi}|(t-\tau)^{-\frac{N}{2}\left(\frac{1}{2}-\frac{1}{r}\right)-\frac{1}{2}}\|\widetilde{q}(\cdot, \tau)\|_{L^{r^{\prime}}(\omega)} \mathrm{d} \tau \\
& +C T^{1 / 2} \int_{0}^{t}\left|\partial_{t} \widetilde{\varphi}\right|(t-\tau)^{-\frac{N}{2}\left(\frac{1}{2}-\frac{1}{r}\right)-\frac{1}{2}}\|\widetilde{q}(\cdot, \tau)\|_{L^{r^{\prime}}(\omega)} \mathrm{d} \tau
\end{aligned}
$$

Using the definition of $\eta$, it is not difficult to see that

$$
|\widetilde{\varphi}| \leq C T^{-3} \mathrm{e}^{-C s T^{-2}} \quad \forall(x, t) \in Q
$$

and

$$
\frac{\mathrm{e}^{-s \eta}}{t^{7 / 2}(T-t)^{7 / 2}} \leq C T^{-7} \mathrm{e}^{-C s T^{-2}} \quad \forall(x, t) \in Q
$$

for $s \geq \sigma(\Omega, \omega, a) T^{2}$. Moreover, using (135) and (145), we can write that

$$
\left|\partial_{t} \widetilde{\varphi}\right|=\left|\frac{\mathrm{e}^{-s \eta}(T-2 t)}{t^{7 / 2}(T-t)^{7 / 2}}\left(s+\frac{3}{2} t T-\frac{3}{2} T^{2}\right)\right| \leq C T^{-6}\left(s+T^{2}\right) \mathrm{e}^{-C s T^{-2}}
$$

for $s \geq \sigma(\Omega, \omega, a) T^{2}$. Thanks to (145) and (146), from (143) we have

$$
\begin{aligned}
\|\widetilde{w}(\cdot, t)\|_{L^{2}(\widetilde{\omega}) \leq} & C T^{-3}\left(1+T^{1 / 2}+T^{1 / 2}\|b\|_{\infty}\right) \mathrm{e}^{-C s T^{-2}} \int_{0}^{t}(t-\tau)^{-\frac{N}{2}\left(\frac{1}{2}-\frac{1}{r}\right)-\frac{1}{2}}\|\widetilde{q}(\cdot, \tau)\|_{L^{r^{\prime}}(\omega)} \mathrm{d} \tau \\
& +C T^{1 / 2} T^{-6}\left(s+T^{2}\right) \mathrm{e}^{-C s T^{-2}} \int_{0}^{t}(t-\tau)^{-\frac{N}{2}\left(\frac{1}{2}-\frac{1}{r}\right)-\frac{1}{2}}\|\widetilde{q}(\cdot, \tau)\|_{L^{r^{\prime}}(\omega)} \mathrm{d} \tau
\end{aligned}
$$

for $s \geq \sigma(\Omega, \omega, a) T^{2}$. If $r^{\prime}$ is such that

$$
\frac{N}{2}\left(\frac{1}{2}-\frac{1}{r}\right)+\frac{1}{2}+\frac{1}{r^{\prime}}<\frac{3}{2}
$$


that is to say

$$
r^{\prime}>\frac{2(N+2)}{N+4}
$$

then, we can apply Young's inequality to $(143)$ and estimate the $L^{2}\left(0, T ; L^{2}(\widetilde{\omega})\right)$-norm of $\widetilde{w}$ as follows:

$$
\begin{aligned}
\|\widetilde{w}\|_{L^{2}\left(0, T ; L^{2}(\widetilde{\omega})\right) \leq} & C T^{\kappa} T^{-3}\left(1+T^{1 / 2}+T^{1 / 2}\|b\|_{\infty}\right) \mathrm{e}^{-C s T^{-2}}\left(\iint_{\omega \times(0, T)}|q|^{r^{\prime}} \mathrm{d} x \mathrm{~d} t\right)^{1 / r^{\prime}} \\
& +C T^{\kappa} T^{1 / 2} T^{-6}\left(s+T^{2}\right) \mathrm{e}^{-C s T^{-2}}\left(\iint_{\omega \times(0, T)}|q|^{r^{\prime}} \mathrm{d} x \mathrm{~d} t\right)^{1 / r^{\prime}}
\end{aligned}
$$

where $C$ is a new positive constant depending on $\Omega, \omega, \widetilde{\omega} r^{\prime}$ and $N, \kappa$ depends on $N$ and $r$ and $s \geq \sigma(\Omega, \omega, a) T^{2}$. Notice that for $N<4$ and $r^{\prime}$ as in (4) the condition (148) is satisfied. For $N \geq 4$ we apply again this process in order to obtain the inequality (133) for $r^{\prime}$ sufficiently small, in such a way that (4) holds for $s \geq \sigma(\Omega, \omega, a) T^{2}$ and with $\alpha$ and $\gamma$ only depending on $N$ (cf. [13]). This ends the proof of Lemma 4.3.

Proof of Proposition 4.2. We will use a global Carleman inequality (46) from Theorem 3.3, some estimates for the weight functions, classical parabolic estimates and technical result of Lemma 4.3.

Step 1: Let $b$ and $q_{T}$ be given and let $q$ be the solution to (118). We will first see that

$$
\iint_{\Omega \times(T / 4,3 T / 4)}|q|^{2} \mathrm{~d} x \mathrm{~d} t \leq T^{6} \exp \left[C\left(1+\frac{1}{T}+\|b\|_{\infty}^{2 / 3}\right)\right] \iint_{\omega \times(0, T)} \mathrm{e}^{-2 s \eta} t^{-3}(T-t)^{-3}|q|^{2} \mathrm{~d} x \mathrm{~d} t .
$$

Let us consider (122), that is true for $s \geq s_{7}$ with $s_{7}$ given by (123). Then, using (125), we obtain that

$$
\iint_{\Omega \times(T / 4,3 T / 4)}|q|^{2} \mathrm{~d} x \mathrm{~d} t \leq C T^{6} \exp \left(C s T^{-2}\right) \iint_{\omega \times(0, T)} \mathrm{e}^{-2 s \eta} t^{-3}(T-t)^{-3}|q|^{2} \mathrm{~d} x \mathrm{~d} t
$$

for all $s \geq s_{8}$. Thanks to the structure of the constant $s_{9}$, given by (126), the inequality (151) written for $s=s_{9}$ implies that (150) is satisfied for any solution $q$ of (118).

Step 2: Let us now prove the first part of desired observability estimate (131), i.e. the following inequality:

$$
\|q(x, 0)\|_{L^{2}(\Omega)}^{2} \leq \exp \left[C H\left(T,\|b\|_{\infty}\right)\right]\left(\iint_{\omega \times(0, T)}|q|^{r^{\prime}} \mathrm{d} x \mathrm{~d} t\right)^{2 / r^{\prime}}
$$

with $H\left(T,\|b\|_{\infty}\right)$ given by $(132)$. Let $\widetilde{\omega}$ be a nonempty open set such that $\widetilde{\omega} \subset \subset \omega \cap \Omega_{0}$. We consider (130), that is to say

$$
\|q(x, 0)\|_{L^{2}(\Omega)}^{2} \leq \frac{2 C}{T} \exp \left(\frac{3 T}{2}\|b\|_{\infty}\right) \iint_{\Omega \times(T / 4,3 T / 4)}|q|^{2} \mathrm{~d} x \mathrm{~d} t .
$$

We apply to the right hand side of this inequality, the estimates (150) written for $\widetilde{\omega}$ and we obtain

$$
\|q(x, 0)\|_{L^{2}(\Omega)}^{2} \leq C T^{5} \exp \left(1+\frac{1}{T}+T\|b\|_{\infty}+\|b\|_{\infty}^{2 / 3}\right) \iint_{\widetilde{\omega} \times(0, T)} \mathrm{e}^{-2 s \eta} t^{-3}(T-t)^{-3}|q|^{2} \mathrm{~d} x \mathrm{~d} t .
$$


On the other hand, we notice that the constant $\sigma(\Omega, \omega, a) T^{2}$ appearing in Lemma 4.3 is less then the constant $s_{9}$ given by (126). So, we can write technical estimate (133) for $s=s_{9}$. This gives

$$
\iint_{\widetilde{\omega} \times(0, T)} \mathrm{e}^{-2 s \eta} t^{-3}(T-t)^{-3}|q|^{2} \mathrm{~d} x \mathrm{~d} t \leq C T^{-3} K_{1}\left(T,\|b\|_{\infty}\right)\left(\iint_{\omega \times(0, T)}|q|^{r^{\prime}} \mathrm{d} x \mathrm{~d} t\right)^{2 / r^{\prime}},
$$

where

$$
K_{1}\left(T,\|b\|_{\infty}\right)=\exp \left(1+\frac{1}{T}+T\|b\|_{\infty}+\|b\|_{\infty}^{2 / 3}\right) T^{\alpha}\left[1+T^{1 / 2}\left(1+\|b\|_{\infty}+\frac{1}{T^{2}}+\frac{1}{T}\|b\|_{\infty}^{2 / 3}\right)\right]^{\gamma} .
$$

Combining (153) and (154), we deduce that

$$
\|q(x, 0)\|_{L^{2}(\Omega)}^{2} \leq C T^{2} K_{1}\left(T,\|b\|_{\infty}\right)\left(\iint_{\omega \times(0, T)}|q|^{r^{\prime}} \mathrm{d} x \mathrm{~d} t\right)^{2 / r^{\prime}}
$$

Consequently, we also have (152).

Step 3: Let us finally deduce that

$$
\iint_{\Omega \times(0, T)} \mathrm{e}^{-2 s \widetilde{C} T^{-1} /(T-t)}(T-t)^{-3}|q|^{2} \mathrm{~d} x \mathrm{~d} t \leq \exp \left[C H\left(T,\|b\|_{\infty}\right)\right]\left(\iint_{\omega \times(0, T)}|q|^{r^{\prime}} \mathrm{d} x \mathrm{~d} t\right)^{2 / r^{\prime}},
$$

where $H\left(T,\|b\|_{\infty}\right)$ is done by (132). Integrating (129) with respect to time and using that $\theta=1$ in $[0, T / 4]$, we obtain for all $t \in[0,3 T / 4]$

$$
\exp \left(2|| b \|_{\infty} t\right) \int_{\Omega}|\theta q(x, t)|^{2} \mathrm{~d} x \leq\left.\int_{T / 4}^{3 T / 4} 2 \exp \left(2\|b\|_{\infty} \tau\right) \int_{\Omega} \theta\left|\partial_{t} \theta \|\right| q\right|^{2} \mathrm{~d} x \mathrm{~d} \tau
$$

Since $\theta \leq 1,\left|\partial_{t} \theta\right|=\left|\partial_{t} \theta_{0}(t / T) / T\right| \leq C / T$, from (158) we obtain

$$
\int_{\Omega}|q(x, t)|^{2} \mathrm{~d} x \leq \frac{2}{T} \exp \left(\frac{3 T}{2}\|b\|_{\infty}\right) \iint_{\Omega \times(T / 4,3 T / 4)}|q|^{2} \mathrm{~d} x \mathrm{~d} t
$$

for all $t \in[0, T / 4]$. Then, we have

$$
\iint_{\Omega \times(0, T / 4)}|q(x, t)|^{2} \mathrm{~d} x \leq \exp \left(\frac{3 T}{2}\|b\|_{\infty}\right) \iint_{\Omega \times(T / 4,3 T / 4)}|q|^{2} \mathrm{~d} x \mathrm{~d} t .
$$

On the other hand, using the definition (44) of the function $\eta$, we can say that there exist a positive constants $C$ and $\widetilde{C}$, depending on $\Omega, \omega$ and $a$, such that

$$
\mathrm{e}^{-2 s \eta} t^{-3}(T-t)^{-3} \geq C T^{-3}(T-t)^{-3} \mathrm{e}^{-2 s \widetilde{C} T^{-1} /(T-t)} \quad \forall x \in \Omega, \quad \forall t \in[T / 4, T]
$$


whenever $s \geq \sigma(\Omega, \omega, a) T^{2}$. We can write

$$
\begin{aligned}
& \iint_{\Omega \times(0, T)} \mathrm{e}^{-2 s \widetilde{C} T^{-1} /(T-t)}(T-t)^{-3}|q|^{2} \mathrm{~d} x \mathrm{~d} t \\
& \leq \iint_{\Omega \times(0, T / 4)} \mathrm{e}^{-2 s \widetilde{C} T^{-1} /(T-t)}(T-t)^{-3}|q|^{2} \mathrm{~d} x \mathrm{~d} t+C T^{3} \iint_{\Omega \times(T / 4, T)} \mathrm{e}^{-2 s \eta} t^{-3}(T-t)^{-3}|q|^{2} \mathrm{~d} x \mathrm{~d} t \\
& \leq T^{-3} \mathrm{e}^{-2 C s T^{-2}} \iint_{\Omega \times(0, T / 4)}|q|^{2} \mathrm{~d} x \mathrm{~d} t+C T^{3} \iint_{\Omega \times(T / 4, T)} \mathrm{e}^{-2 s \eta} t^{-3}(T-t)^{-3}|q|^{2} \mathrm{~d} x \mathrm{~d} t \\
& \leq T^{-3} \mathrm{e}^{C\left(-s T^{-2}+T\|b\|_{\infty}\right)} \iint_{\Omega \times(T / 4,3 T / 4)}|q|^{2} \mathrm{~d} x \mathrm{~d} t \\
& +C T^{3} \iint_{\Omega \times(0, T)} \mathrm{e}^{-2 s \eta} t^{-3}(T-t)^{-3}|q|^{2} \mathrm{~d} x \mathrm{~d} t
\end{aligned}
$$

Here, we have used (161) and (160). Applying (151), written for $\widetilde{\omega}$ to the first term of the right hand side of (161) and Carleman inequatily (122) (written also for $\widetilde{\omega}$ ) to the second one, we deduce that for $s=s_{9}$ the following holds:

$$
\begin{aligned}
& \iint_{Q} \mathrm{e}^{-2 s \widetilde{C} T^{-1} /(T-t)}(T-t)^{-3}|q|^{2} \mathrm{~d} x \mathrm{~d} t \\
& \leq C T^{3}\left(1+\exp \left[C\left(s T^{-2}+T\|b\|_{\infty}\right)\right]\right) \iint_{\widetilde{\omega} \times(0, T)} \mathrm{e}^{-2 s \eta} t^{-3}(T-t)^{-3}|q|^{2} \mathrm{~d} x \mathrm{~d} t \\
& \leq C T^{3} \exp \left[C\left(1+\frac{1}{T}+T\|b\|_{\infty}+\|b\|_{\infty}^{2 / 3}\right)\right] \iint_{\widetilde{\omega} \times(0, T)} \mathrm{e}^{-2 s \eta} t^{-3}(T-t)^{-3}|q|^{2} \mathrm{~d} x \mathrm{~d} t
\end{aligned}
$$

Using (154) in (163), we get (157). This ends the proof of Proposition 4.2.

\section{Proof of Theorem 2.1}

We will first prove a null controllability result for a corresponding linear problem which is interesting for itself. For this proof we use a result of approximate controllability to the zero state and then apply an observability inequality to obtain estimates which enable us to pass to the limit. We could then try to use this null controllability result and apply the fixed point method to treat the nonlinear problem. For technical reasons, we prefer here to apply the fixed point method to the approximate controllability problem to the zero state and then use the observability inequality to obtain the desired result of exact controllability to the trajectories for the nonlinear system.

\subsection{A null controllability result for a linear problem}

We will consider the linear system

$$
\left\{\begin{array}{lr}
\partial_{t} y-\operatorname{div}(a(x) \nabla y)+b y=v 1_{\omega}+k & \text { in } Q \\
y=0 & \text { on } \Sigma \\
y(x, 0)=y_{0} & \text { in } \Omega
\end{array}\right.
$$


where $a$ satisfies $(5,6,9), b \in L^{\infty}(Q), k \in L^{r}\left(0, T ; L^{r}(\Omega)\right)$, and $y_{0} \in L^{2}(\Omega)$ are given. The following holds:

Theorem 5.1. Let $T>0$. Assume that $\omega \cap \Omega_{0} \neq \emptyset$, Condition 2.1 (resp. Condition 2.2) in case (1) (resp. in case (2)) are fulfilled and a satisfies $(5,6,9)$ and (10). We take data $b \in L^{\infty}(Q), y_{0} \in L^{2}(\Omega), k \in L^{2}(Q)$ and such that

$$
\iint_{Q} \mathrm{e}^{2 s \widetilde{C} T^{-1} /(T-t)}(T-t)^{3}|k|^{2} \mathrm{~d} x \mathrm{~d} t<+\infty
$$

for $s \geq \sigma(\Omega, \omega, a)\left(T+T^{2}+T^{2}\|b\|_{\infty}^{2 / 3}\right)$. Then for each case (1) or (2), there exists a control $\widehat{v} \in L^{r}(\mathcal{O} \times(0, T))$ such that the corresponding solution $\widehat{y}$ of (164) verifies

$$
\widehat{y}(x, T)=0 \quad \text { in } \Omega .
$$

Moreover, $\widehat{v}$ can be chosen satisfying the estimate

$$
\|\widehat{v}\|_{L^{r}(\omega \times(0, T))} \leq \widehat{H}\left(\Omega, \omega, a, T,\|b\|_{\infty}\right)\left(\left\|y_{0}\right\|_{L^{2}(\Omega)}+\left\|\mathrm{e}^{s \widetilde{C} T^{-1} /(T-t)}(T-t)^{3 / 2} k\right\|_{L^{2}(Q)}\right),
$$

with

$$
\widehat{H}\left(\Omega, \omega, a, T,\|b\|_{\infty}\right)=\exp \left[C\left(1+\frac{1}{T}+T+\left(T+T^{1 / 2}\right)\|b\|_{\infty}+\|b\|_{\infty}^{2 / 3}\right)\right] .
$$

Remark 5.1. Notice that if $k \in L^{r}\left(0, T ; L^{r}(\Omega)\right)$ such that (165) holds, then $k$ vanishes exponentially at $t=T$. If $k=0$ in a neighbourhood of $t=T$ and $k \in L^{r}\left(0, T ; L^{r}(\Omega)\right)$ then (165) is satisfied.

Proof of Theorem 5.1. Let us fix $T>0, b \in L^{\infty}(Q), y_{0} \in L^{2}(\Omega), k \in L^{r}\left(0, T ; L^{r}(\Omega)\right)$, with $r$ verifying (4) and such that (165) holds. For every $\varepsilon>0$, let us consider the functional $J_{\varepsilon}$ definite by

$$
J_{\varepsilon}\left(q_{T}\right)=\frac{1}{2}\left(\iint_{\omega \times(0, T)}|q|^{r^{\prime}} \mathrm{d} x \mathrm{~d} t\right)^{2 / r^{\prime}}+\varepsilon\left\|q_{T}\right\|_{L^{2}(\Omega)}+\int_{\Omega} q(x, 0) y_{0}(x) \mathrm{d} x+\iint_{Q} k q \mathrm{~d} x \mathrm{~d} t \quad \forall q_{T} \in L^{2}(\Omega),
$$

where $q$ is the solution of (118) associated to $q_{T} \in L^{2}(\Omega)$ and $r^{\prime}$ is the dual exponent of $r$. It is easy to see that $J_{\varepsilon}$ is a continuous and strictly convex functional. Furthermore, from (122), it is immediate to deduce the following unique continuation property for the adjoint problem (118):

$$
\text { If } q=0 \text { in } \omega \times(0, T) \text {, then } q \equiv 0 \text {. }
$$

Then, arguing as in [9] and [10], we see that

$$
\liminf _{\left\|q_{T}\right\|_{L^{2}} \rightarrow \infty} \frac{J_{\varepsilon}\left(q_{T}\right)}{\left\|q_{T}\right\|_{L^{2}}} \geq \varepsilon
$$

and, therefore, $J_{\varepsilon}$ achieves its minimum at a unique point $\widehat{q}_{T, \varepsilon} \in L^{2}(\Omega)$. Let $\widehat{q}_{\varepsilon}$ be the solution of (118) associated to $\widehat{q}_{T, \varepsilon}$. Arguing again as in [9], we take in (164) $v=\widehat{v}_{\varepsilon}$, where

$$
\widehat{v}_{\varepsilon}=\operatorname{sgn}\left(\widehat{q}_{\varepsilon}\right)\left|\widehat{q}_{\varepsilon}\right|^{r^{\prime}-1}\left\|\widehat{q}_{\varepsilon}\right\|_{L^{r^{\prime}}(\omega \times(0, T))} 1_{\omega},
$$

then, we find a solution $\widehat{y}_{\varepsilon}$ satisfying

$$
\left\|\widehat{y}_{\varepsilon}(\cdot, T)\right\|_{L^{2}(\Omega)} \leq \varepsilon
$$


Moreover, we can obtain the estimates of the $L^{r}(\omega \times(0, T))$-norm of the control independent of $\varepsilon$. We claim that for a suitable $C=C(\Omega, \omega, a)>0$ we have:

$$
\left\|\widehat{v}_{\varepsilon}\right\|_{L^{r}(\omega \times(0, T))} \leq \widehat{H}\left(\Omega, \omega, T, a,\|b\|_{\infty}\right)\left(\left\|y_{0}\right\|_{L^{2}(\Omega)}+\left\|\mathrm{e}^{s \widetilde{C} T^{-1} /(T-t)}(T-t)^{3 / 2} k\right\|_{L^{2}(Q)}\right)
$$

for all $\varepsilon>0$ and with $\widehat{H}$ given by (168). Indeed, from (170) we can write that

$$
\left\|\widehat{v}_{\varepsilon}\right\|_{L^{r}(\omega \times(0, T))}=\left(\iint_{\omega \times(0, T)}\left|\widehat{q}_{\varepsilon}\right|^{r^{\prime}} \mathrm{d} x \mathrm{~d} t\right)^{1 / r^{\prime}}
$$

On the other hand, at the minimum $\widehat{q}_{T, \varepsilon}$, we have

$$
J_{\varepsilon}\left(\widehat{q}_{T, \varepsilon}\right) \leq J_{\varepsilon}(0)=0
$$

Taking (169) into account, we see that

$$
\begin{aligned}
\frac{1}{2}\left(\iint_{\omega \times(0, T)}\left|\widehat{q}_{\varepsilon}\right|^{\prime^{\prime}} \mathrm{d} x \mathrm{~d} t\right)^{2 / r^{\prime}} \leq-\int_{\Omega} \widehat{q}_{\varepsilon}(x, 0) y_{0}(x) \mathrm{d} x-\iint_{Q} k q \mathrm{~d} x \mathrm{~d} t \leq\left\|\widehat{q}_{\varepsilon}(\cdot, 0)\right\| L_{L^{2}(\Omega)}\left\|y_{0}(x)\right\|_{L^{2}(\Omega)} \\
+\left(\iint_{Q} \mathrm{e}^{2 s \widetilde{C} T^{-1} /(T-t)}(T-t)^{3}|k|^{2} \mathrm{~d} x \mathrm{~d} t\right)^{1 / 2}\left(\iint_{Q} \mathrm{e}^{-2 s \widetilde{C} T^{-1} /(T-t)}(T-t)^{-3}|q|^{2} \mathrm{~d} x \mathrm{~d} t\right)^{1 / 2}
\end{aligned}
$$

In view of (131) and (165), the estimate (172) holds.

Since $\widehat{v}_{\varepsilon}$ is uniformly bounded in $L^{r}(\omega \times(0, T))$, for an appropriate subsequence, we deduce that

$$
\widehat{v}_{\varepsilon} \rightarrow \widehat{v} \quad \text { weakly in } L^{r}(\omega \times(0, T))
$$

where $\widehat{v} \in L^{r}(\omega \times(0, T))$ and satisfies (167). Accordingly,

$$
\widehat{y}_{\varepsilon}(T) \rightarrow \widehat{y}(T) \quad \text { in } L^{2}(\Omega)
$$

where $\widehat{y}$ is the solution of (164) associated to $\widehat{v}$. Since we have (171) for all $\varepsilon>0$, we also have (166). This ends the proof of Theorem 5.1.

Remark 5.2. Notice that, using the argument of [13] and [9], it is also possible to obtain the controls in $L^{\infty}\left(0, T ; L^{\infty}(\omega)\right)$.

\subsection{The fixed point method. Conclusion}

We are now ready to complete the proof of Theorem 2.1. We will apply a fixed point method to obtain $\varepsilon$-approximate controllability to the zero state for the nonlinear transmission problem, using the ideas of [9] and [10]. Then the main ingredients of our proof are the explicit estimates of the controls needed for controllability of a linear transmission problem, which have been obtained in the previous section and the choice of the control time depending on the size of the potential. This will allow us to pass to the limit in the nonlinear problem when $\varepsilon$ tends to zero and obtain the complete results of Theorem 2.1. 
Step 1: Let us consider a trajectory $y^{*}$, solution of the problem (12) without control. We introduce the change of variable $p=y-y^{*}$, where $y$ is a solution of (8). Then, we obtain that

$$
\left\{\begin{array}{lr}
\partial_{t} p-\operatorname{div}(a(x) \nabla p)+f\left(y^{*}+p\right)-f\left(y^{*}\right)=v 1_{\omega} & \text { in } Q \\
p=0 & \text { on } \Sigma \\
p(x, 0)=p_{0} & \text { in } \Omega
\end{array}\right.
$$

where $p_{0}=y_{0}-y_{0}^{*}$. Theorem 2.1 will be proved if we show that, for each $p_{0} \in L^{2}(\Omega)$, there exists $v \in$ $L^{r}(\omega \times(0, T))$ such that

$$
p(x, T)=0 \text { in } \Omega \text {. }
$$

We will first consider the case in which $p_{0} \in L^{\infty}$ and $f \in C^{1}$ in $\mathbb{R}$. We denote by $h$ the following function:

$$
h(a, s)=\left\{\begin{array}{cl}
\frac{f(a+s)-f(a)}{s} & \text { if } s \neq 0, \\
f^{\prime}(a) & \text { if } s=0 .
\end{array}\right.
$$

Then $h$ is continuous. Thanks to hypothesis (11) we know that for each $\eta>0$, there exists $C_{\eta}>0$ (depending only of $\eta$ and the function $f$ ) such that

$$
\left|h\left(y^{*}(x, t), s\right)\right|^{2 / 3} \leq C_{\eta}+\eta \log (1+|s|) \quad \forall s \in \mathbb{R}, \quad \forall(x, t) \in Q .
$$

Step 2: Let $\eta>0$ and $R>0$ be given positive constants whose values will be fixed later on.

Let us fix a time

$$
T^{R}=\min \left\{T,\|h\|_{L^{\infty}(-R, R)}^{-2 / 3},\|h\|_{L^{\infty}(-R, R)}^{-1 / 3}\right\}
$$

For simplicity, in the sequel, we will refer only to the case (1), but we also take into account the dependence of the constants corresponding to the case (2).

Step 3: (a) We consider the truncation function $\mathbf{T}_{R}: \mathbb{R} \mapsto \mathbb{R}$, which is given as follows:

$$
\mathbf{T}_{R}(s)=\left\{\begin{array}{cc}
s & \text { if }|s| \leq R \\
R \operatorname{sgn}(s) & \text { otherwise. }
\end{array}\right.
$$

For each $z \in L^{2}(Q)$, we consider the linear system

$$
\begin{cases}\partial_{t} p-\operatorname{div}(a(x) \nabla p)+h\left(y^{*}(x, t), \mathbf{T}_{R}(z)\right) p=v 1_{\omega} & \text { in } \Omega \times\left(0, T^{R}\right), \\ p=0 & \text { on } \Gamma \times\left(0, T^{R}\right), \\ p(x, 0)=p_{0} & \text { in } \Omega .\end{cases}
$$

Notice that (180) is of the form (164), with $b=h\left(y^{*}, \mathbf{T}_{R}(z)\right) \in L^{\infty}(Q)$. Then we can apply the arguments of the proof of Theorem 5.1 to (180). In fact, we will apply this result in a time interval $\left(0, T^{R}\right)$, where $T^{R}$ is given by (179). This is a key point in this proof that will drive to appropriate estimates (the idea is taken from [13] and it has been applied later in $[8]$ ).

(b) More precisely, for every $\varepsilon>0$, let us consider the functional $J_{\varepsilon}$ of the form (169). Arguing as in the proof of Theorem 5.1, we obtain the existence of a control $v_{z}^{\varepsilon} \in L^{r}\left(\omega \times\left(0, T^{R}\right)\right)$, minimizing the $L^{r}\left(\omega \times\left(0, T^{R}\right)\right)$ norm of the form $v_{z}^{\varepsilon}=\operatorname{sgn}\left(q_{z}^{\varepsilon}\right)\left|q_{z}^{\varepsilon}\right|^{r^{\prime}-1}\left\|q_{z}^{\varepsilon}\right\|_{L^{r^{\prime}(\omega \times(0, T))}} 1_{\omega},\left(r^{\prime}>1\right)$ with $q_{z}^{\varepsilon}$ the solution of the corresponding 
problem (118), such that the solution $p_{z}^{\varepsilon}$ of (180) with $v=v_{z}^{\varepsilon}$ satisfies

$$
\left\|p_{z}^{\varepsilon}\left(\cdot, T^{R}\right)\right\|_{L^{2}(\Omega)} \leq \varepsilon .
$$

Then, from (172) we have

$$
\left\|v_{z}^{\varepsilon}\right\|_{L^{r}\left(\omega \times\left(0, T^{R}\right)\right)} \leq C_{1}\left(\Omega, \omega, a, T^{R},\|h\|_{L^{\infty}(-R, R)}\right)|| p_{0} \|_{L^{2}(\Omega)}
$$

where

$$
C_{1}\left(\Omega, \omega, a, T^{R},\|h\|_{L^{\infty}(-R, R)}\right)=\mathrm{e}^{C(\Omega, \omega, a)\left(1+\frac{1}{T^{R}}+T^{R}+\left(T^{R}+T^{R 1 / 2}\right)\|h\|_{L^{\infty}(-R, R)}+\|h\|_{L^{\infty}(-R, R)}^{2 / 3}\right)} .
$$

Using now the definition of $T^{R}$, we deduce

$$
\left\|v_{z}^{\varepsilon}\right\|_{L^{r}\left(\omega \times\left(0, T^{R}\right)\right)} \leq \mathrm{e}^{C_{2}(\Omega, \omega, a, T)\left(1+\|h\|_{L^{\infty}(-R, R)}^{2 / 3}\right)}\left\|p_{0}\right\|_{L^{2}(\Omega)} .
$$

Moreover, thanks to (178), from (184) for each $z \in L^{2}(Q)$ we obtain the following:

$$
\begin{aligned}
\left\|v_{z}^{\varepsilon}\right\|_{L^{r}\left(\omega \times\left(0, T^{R}\right)\right)} & \leq \mathrm{e}^{C_{2}(\Omega, \omega, a, T)\left(1+C_{\eta}+\eta \log (1+R)\right)}\left\|p_{0}\right\|_{L^{2}(\Omega)} \\
& =C_{3}\left(\Omega, \omega, a, T, \eta, p_{0}\right)(1+R)^{\eta C_{2}(\Omega, \omega, a, T)} .
\end{aligned}
$$

Here we have used the fact that from (178) we can easily write that

$$
\|h\|_{L^{\infty}(-R, R)}^{2 / 3} \leq C_{\eta}+\eta \log (1+|R|) .
$$

(c) For each $\varepsilon>0$ and $z \in L^{2}(Q)$ we introduce the mapping $\Lambda: L^{2}(Q) \mapsto L^{2}(Q)$ defined as follows: for each $z \in L^{2}(Q), \Lambda(z)=p_{z}^{\varepsilon}$, where $p_{z}^{\varepsilon}$ is the the solution of (180) satisfying (181) with $v=v_{z}^{\varepsilon}$ constructed in the point (b) of this step. In fact, $\Lambda$ is of the following form

$$
z \in L^{2}(Q) \mapsto \mathbf{T}_{R}(z) \in L^{\infty}(Q) \mapsto h\left(y^{*}, \mathbf{T}_{R}(z)\right) \in L^{\infty}(Q) \mapsto v_{z}^{\varepsilon} \in L^{r}\left(\omega \times\left(0, T^{R}\right)\right) \mapsto p_{z}^{\varepsilon} \in L^{2}(Q) .
$$

Arguing as in [9], we apply Schauder's theorem and we deduce for each $\varepsilon>0$ the existence of a fixed point $p^{\varepsilon}$ (associated to $v^{\varepsilon}$ ) of $\Lambda$ which verifies

$$
\left\|p_{z}\left(\cdot, T^{R}\right)\right\|_{L^{2}(\Omega)} \leq \varepsilon
$$

Notice that we have used that the solution $p_{z}^{\varepsilon}$ of (180) is bounded (uniformly in $z$ ) in $L^{2}\left(0, T^{R} ; H_{0}^{1}(\Omega)\right.$ ) and its time derivative $\partial_{t} p_{z}^{\varepsilon}$ is bounded in $L^{2}\left(0, T^{R} ; H^{-1}(\Omega)\right)$.

(d) Let $p^{\varepsilon}$ be a fixed point of $\Lambda$ associated to the control $v^{\varepsilon}$ constructed as above. Since (185) holds for $v^{\varepsilon}$, then $v^{\varepsilon}$ is bounded in $L^{r}\left(\omega \times\left(0, T^{R}\right)\right)$ uniformly in $\varepsilon, p^{\varepsilon}$ is bounded in $L^{2}\left(0, T^{R} ; H_{0}^{1}(\Omega)\right)$ and $\partial_{t} p_{z}^{\varepsilon}$ is bounded in $L^{2}\left(0, T^{R} ; H^{-1}(\Omega)\right)$. For an appropriate subsequence, we deduce that as $\varepsilon \rightarrow 0$

$$
v^{\varepsilon} \rightarrow \bar{v}^{R} \quad \text { weakly in } L^{r}\left(\omega \times\left(0, T^{R}\right)\right),
$$

where $\bar{v}^{R} \in L^{r}\left(\omega \times\left(0, T^{R}\right)\right)$ also satisfies (185), and

$$
\begin{aligned}
& p^{\varepsilon} \rightarrow \bar{p}^{R} \quad \text { weakly in } L^{2}\left(0, T^{R} ; H_{0}^{1}(\Omega)\right), \\
& \partial_{t} p^{\varepsilon} \rightarrow \partial_{t} \bar{p}^{R} \quad \text { weakly in } L^{2}\left(0, T^{R} ; H^{-1}(\Omega)\right),
\end{aligned}
$$


where $\bar{p}^{R}$ is the solution of the following problem:

$$
\begin{cases}\partial_{t} \bar{p}^{R}-\operatorname{div}\left(a(x) \nabla \bar{p}^{R}\right)+h\left(y^{*}(x, t), \mathbf{T}_{R}\left(\bar{p}^{R}\right)\right) \bar{p}^{R}=\bar{v}^{R} 1_{\omega} & \text { in } \Omega \times\left(0, T^{R}\right), \\ \bar{p}^{R}=0 & \text { on } \Gamma \times\left(0, T^{R}\right), \\ \bar{p}^{R}(x, 0)=p_{0} & \text { in } \Omega .\end{cases}
$$

Then

$$
p^{\varepsilon}\left(T^{R}\right) \rightarrow \bar{p}^{R}\left(T^{R}\right) \quad \text { in } L^{2}(\Omega)
$$

and since we have (187) for all $\varepsilon>0$, we also have

$$
\bar{p}^{R}\left(T^{R}\right)=0
$$

On the other hand, since $\bar{v}^{R} \in L^{r}\left(0, T^{R} ; L^{r}(\omega)\right)$, with $r$ as in (4), we can write (cf. for example [2] and [3]) that

$$
\left\|\bar{p}^{R}\right\|_{\infty} \leq \mathrm{e}^{T^{R}\left\|h\left(y^{*}, \mathbf{T}_{R}\left(\bar{p}^{R}\right)\right)\right\|_{\infty}}\left\|p_{0}\right\|_{\infty}+T^{R} \mathrm{e}^{T^{R}\left\|h\left(y^{*}, \mathbf{T}_{R}\left(\bar{p}^{R}\right)\right)\right\|_{\infty}}\left\|\bar{v}^{R}\right\|_{L^{r}\left(0, T^{R} ; L^{r}(\omega)\right)} .
$$

Using again the definition of $T^{R}$ and also taking into account (185) and (186), we deduce from (191) that $\bar{p}^{R}$ verifies

$$
\begin{aligned}
\left\|\bar{p}^{R}\right\|_{\infty} & \leq \mathrm{e}^{C_{4}(\Omega, \omega, a, T)\left(1+\|h\|_{L^{\infty}(-R, R)}^{2 / 3}\right)}\left(\left\|p_{0}\right\|_{\infty}+\left\|\bar{v}^{R}\right\|_{L^{r}\left(0, T^{R} ; L^{r}(\omega)\right)}\right) \\
& \leq C_{5}\left(\Omega, \omega, a, T, \eta, p_{0}\right)(1+R)^{\eta C_{6}(\Omega, \omega, a, T)}
\end{aligned}
$$

Notice that in (192) the constant $C_{5}$ is independent of $R$ and the constant $C_{6}$ is independent of $\eta$ and $R$. Let us extend by zero $\bar{p}^{R}$ and $\bar{v}^{R}$ to the whole cylinder $Q=\Omega \times(0, T)$ and for simplicity, we still call them $\bar{p}^{R}$ and $\bar{v}^{R}$. It is clear that (192) holds and that $\bar{v}^{R}$ is such that

$$
\bar{p}^{R}(T)=0 .
$$

(e) In order to conclude the proof of this theorem, it is sufficient to check that for $\eta$ and $R$ suitably chosen, $\bar{p}^{R}$ (defined on $\Omega \times(0, T)$ ) satisfies

$$
\left\|\bar{p}^{R}\right\|_{\infty} \leq R
$$

Then we can say that $\mathbf{T}_{R}\left(\bar{p}^{R}\right)=\bar{p}^{R}$. Of course, this implies the existence of a control $v \in L^{r}\left(0, T ; L^{r}(\omega)\right)$ such that the solution of (176) satisfies (177). Indeed, from (192) we can choose $\eta=1 /\left(2 C_{6}\right)$ and $R>0$ such that

$$
C_{5}\left(\Omega, \omega, a, T, p_{0}\right)(1+R)^{\eta C_{6}(\Omega, \omega, a, T)}<R .
$$

Then we obtain (193). This proves Theorem 2.1 when $p_{0} \in L^{\infty}(\Omega)$ and $f \in C^{1}(\mathbb{R})$. We just mention that we treat the case in which $f$ is only locally Lipschitz continuous as for example in [9] and [13] using approximations of $f$ by $C^{1}$ functions. Then, in this case we deduce the existence of a control $v \in L^{r}\left(0, T ; L^{r}(\omega)\right)$ such that the corresponding solution to (176) verifies (177).

Finally, if $p_{0} \in L^{2}(\Omega)$, for $\delta>0$ sufficiently small we set $v \equiv 0$ for $t \in(0, \delta)$. Using the regularizing effect of the heat equation (see, for example [21] and [22]), we deduce that the corresponding (local) solution $p$ of (176) satisfies $p(\cdot, \delta) \in L^{\infty}(\Omega)$. Then, we argue as above for $p$ in the interval $[\delta, T]$ and we obtain a control $v \in L^{r}\left(0, T ; L^{r}(\omega)\right)$ such that $(177)$ holds. This ends the proof of Theorem 2.1. 


\section{Proofs of Lemma 3.1 and Lemma 3.2}

In this section we will present the construction of the weight functions we used for our global Carleman inequalities.

Proof of the Lemma 3.1. We will proceed in several steps.

Step 1: Let $\zeta$ and $x$ be vector fields verifying Condition 2.1. First, we will construct a function $\widetilde{\beta}_{1} \in C^{1}\left(\bar{\Omega}_{1}\right)$, such that

$$
\begin{aligned}
\widetilde{\beta}_{1} & >0 \text { in } \Omega_{1}, \\
\widetilde{\beta}_{1} & =0 \text { on } \Gamma, \\
\partial_{n} \widetilde{\beta}_{1} & <0 \text { on } \Gamma, \\
\widetilde{\beta}_{1} & =1 \text { on } S, \\
\partial_{n} \widetilde{\beta}_{1} & >0 \text { on } S, \\
\nabla \widetilde{\beta}_{1} & \neq 0 \text { in } \bar{\Omega}_{1} .
\end{aligned}
$$

For $t \in\left[0, t_{1}\left(x_{0}\right)\right]$, let us introduce the following change of variables:

$$
\tau=\frac{t}{t_{1}\left(x_{0}\right)}, \quad \tau \in[0,1], \quad \widetilde{x}(\tau)=x(t) .
$$

Observe that, if we take $x \in \bar{\Omega}_{1}$, then there exists $x_{0} \in \Gamma$ such that $x=x\left(t, x_{0}\right)$ for $t \in\left[0, t_{1}\left(x_{0}\right)\right]$ or, in other words, there exists $\tau \in[0,1]$ such that $\widetilde{x}(0)=x_{0} \in \Gamma, \widetilde{x}(\tau)=x(t)$ and $\widetilde{x}(1)=x\left(t_{1}\left(x_{0}\right)\right) \in S$. Moreover

$$
\frac{\mathrm{d} \widetilde{x}}{\mathrm{~d} \tau}=\frac{\mathrm{d} x}{\mathrm{~d} t} t_{1}\left(x_{0}\right)=\zeta(x(t)) t_{1}\left(x_{0}\right) .
$$

Let us set

$$
\widetilde{\beta}_{1}(x(t))=\widetilde{\beta}_{1}(\widetilde{x}(\tau))=\tau, \quad \tau \in[0,1] .
$$

This function verifies the following properties:

$$
\begin{aligned}
\frac{\mathrm{d} \widetilde{\beta}_{1}}{\mathrm{~d} \tau}(x)=1 & \text { for all } x \in \Omega_{1}, \\
0<\widetilde{\beta}_{1}(x)<1 & \text { for all } x \in \Omega_{1}, \\
\widetilde{\beta}_{1}(x)=0 & \text { for all } x \in \Gamma,
\end{aligned}
$$

and

$$
\widetilde{\beta}_{1}(x)=\widetilde{\beta}_{1}(\widetilde{x}(1))=1 \quad \text { for all } x \in S .
$$

On the other hand, from (200) and (202), for all $x \in \Omega_{1}$, we have

$$
\frac{\mathrm{d} \widetilde{\beta}_{1}}{\mathrm{~d} \tau}(x(\tau))=\nabla \widetilde{\beta}_{1}(\widetilde{x}(\tau)) \frac{\mathrm{d} \widetilde{x}}{\mathrm{~d} \tau}(\tau)=\nabla \widetilde{\beta}_{1}(x) \zeta(x(t)) t_{1}\left(x_{0}\right)=1,
$$

therefore

$$
\nabla \widetilde{\beta}_{1}(x) \neq 0 \quad \text { for all } x \in \Omega_{1} \text {. }
$$


Moreover, using $(200,205)$ and (206), we can write that

$$
\nabla \widetilde{\beta}_{1}(x) \frac{\mathrm{d} \widetilde{x}}{\mathrm{~d} \tau}=\left(\nabla \widetilde{\beta}_{1}(x) \cdot n\right)\left(\frac{\mathrm{d} \widetilde{x}}{\mathrm{~d} \tau} \cdot n\right)=\left(\nabla \widetilde{\beta}_{1}(x) \cdot n\right)(\zeta(x) \cdot n) t_{1}\left(x_{0}\right)=1 \quad \text { for all } x \in S .
$$

Then, taking into account (17), we deduce that

$$
\nabla \widetilde{\beta}_{1}(x) \cdot n>0 \text { for all } x \in S .
$$

This means (198). By the similar way, it is easy to check (196). Thus we also have (199).

In order to obtain $\widetilde{\beta}_{1} \in C^{2}\left(\bar{\Omega}_{1}\right)$, we just notice that we can approximate the function of class $C^{1}$, which we have constructed above by an other function of class $C^{2}$ (that we keep calling $\widetilde{\beta}_{1}$ ), such that it still satisfies the properties (194-199).

Let us now consider the diffusion coefficients $a_{i}, i=0,1$ such that (5) holds and let $\omega_{0} \subset \subset \omega \cap \Omega_{0}$.

Step 2: For $\varepsilon>0$ small enough, we set

$$
U_{\varepsilon}(S)=\left\{x: x \in \Omega_{0}, \quad \operatorname{dist}(x, S)<\varepsilon\right\} .
$$

We can construct a function $\alpha_{0}$ in $\overline{U_{\varepsilon}(S)}$, such that $\alpha_{0} \in C^{2}\left(\overline{U_{\varepsilon}(S)}\right)$ and

$$
\begin{array}{ll}
\alpha_{0}=1 & \text { on } S, \\
\alpha_{0}>0 & \text { in } \overline{U_{\varepsilon}(S)}, \nabla \alpha_{0} \neq 0 \quad \text { on } S, \\
\nabla \alpha_{0} & \text { in } \overline{U_{\varepsilon}(S)}
\end{array}
$$

and

$$
a_{0} \partial_{n} \alpha_{0}=a_{1} \partial_{n} \widetilde{\beta}_{1} \quad \text { on } S \text {. }
$$

Now, we extend this function in $\Omega_{0}$ to a function that we call again $\alpha_{0}$, with $\alpha_{0} \in C^{2}\left(\bar{\Omega}_{0}\right)$ and $\alpha_{0}>0$ in $\Omega_{0}$.

Step 3: Thanks to the Morse theorem, we deduce that there exists a sequence of Morse functions $\theta_{k}, k \geq 1$ (functions with isolated critical points i.e. their gradient vanishes only in a finite number of points), such that

$$
\theta_{k} \rightarrow \alpha_{0} \quad \text { in } C^{2}\left(\bar{\Omega}_{0}\right) \quad \text { if } k \rightarrow+\infty .
$$

If $\theta_{k}$ is close enough to $\alpha_{0}$, the points where $\nabla \theta_{k}$ vanishes can not be in $\overline{U_{\varepsilon}(S)}$. Moreover, we can assume that for some $\delta>0$ we have

$$
\left|\nabla \alpha_{0}\right| \geq \delta>0 \quad \text { in } \overline{U_{\varepsilon}(S)} .
$$

Let us construct a Morse function $\mu \in C^{2}\left(\bar{\Omega}_{0}\right)$, such that

$$
\begin{array}{cc}
\mu=1 \quad \text { on } S, \quad \partial_{n} \mu>0 & \text { on } S \\
a_{0} \partial_{n} \mu=a_{1} \partial_{n} \widetilde{\beta}_{1} & \text { on } S
\end{array}
$$

and

$$
\nabla \mu \neq 0 \quad \text { in } \overline{U_{\varepsilon}(S)}
$$

For this, we consider $\varphi \in \mathcal{D}\left(\overline{U_{\varepsilon}(S)}\right)$ and $\varphi=1$ in $\overline{U_{\varepsilon_{0}}(S)}$, with $0<\varepsilon_{0}<\varepsilon$. We set

$$
\mu_{k}(x)=\theta_{k}(x)+\varphi(x)\left(\alpha_{0}(x)-\theta_{k}(x)\right) .
$$


It is clear that

$$
\mu_{k}=\alpha_{0} \quad \text { in } \overline{U_{\varepsilon_{0}}(S)} .
$$

Then, the function $\mu_{k}$ satisfies (211) and (212). Moreover, we have

$$
\nabla \mu_{k}=\nabla \theta_{k} \quad \text { in } \overline{\Omega_{0} \backslash U_{\varepsilon}(S)}
$$

and

$$
\nabla \mu_{k}=\nabla \theta_{k}+\varphi\left(\nabla \alpha_{0}-\nabla \theta_{k}\right)+\nabla \varphi\left(\alpha_{0}-\theta_{k}\right) \text { in } \overline{U_{\varepsilon}(S)}
$$

Then, using (209) and (210) in (215), we deduce that there exist a positive number $k_{0}=k_{0}(\delta)$ such that, if $k \geq k_{0}$ we have

$$
\left|\nabla \mu_{k}\right| \geq\left|\nabla \theta_{k}\right|-2\|\varphi\|_{C^{1}}\left\|\alpha_{0}-\theta_{k}\right\|_{C^{1}} \geq \frac{\delta}{2} \quad \text { in } \Omega_{0} \cap U_{\varepsilon}(S)
$$

We choose $k \geq k_{0}$ and we set $\mu(x)=\mu_{k}(x)$. Then, $\mu$ is a Morse function whose gradient vanishes only in the set of points where the gradient of $\theta_{k}$ vanishes. This, together with (214), implies that (211) and (213) hold.

Step 4: On the other hand, arguing as in [15], we can deduce that there exists a mapping $g: \Omega_{0} \mapsto \Omega_{0}$ which is a diffeomorphism on $\Omega_{0}$, which leaves invariant $\overline{U_{\varepsilon}(S)}$ and transports the points where the gradient of $\mu$ vanishes in $\omega_{0}$. We set

$$
\widetilde{\beta}_{0}(x)=\mu(g(x)) .
$$

Then, equation (33) holds. Thanks to the properties (211) of the function $\mu$, we also have $(30,31)$ and $(32)$.

This ends the proof of Lemma 3.1 .

Proof of the Lemma 3.2. Assume that we are in the situation of Case (2). Let $\omega_{0} \subset \subset \omega \cap \Omega_{0}$ be an arbitrary fixed open subset of $\Omega_{0}$.

Step 1: We assume that there exist $\mathcal{O}_{1}, \mathcal{O}_{2} \subset \subset \Omega_{1}$ two open disjoint subsets, such that Condition 2.2 holds between $\Omega_{1}$ and each one of two sets $\mathcal{O}_{1}$ and $\mathcal{O}_{2}$. Then, as in the first part of the proof of Lemma 3.1, we construct two functions $\beta_{1}^{i} \in C^{1}\left(\bar{\Omega}_{1} \backslash \mathcal{O}_{i}\right), \beta_{1}^{i}>0$ in $\bar{\Omega}_{1} \backslash \mathcal{O}_{i}, i=1,2$, such that

$$
\begin{array}{ll}
\beta_{1}^{i}=2 \quad \text { on } S, & i=1,2, \\
\partial_{n} \beta_{1}^{i}>0 \text { on } S, & i=1,2, \\
\partial_{n} \beta_{1}^{i}>0 \text { on } \partial \mathcal{O}_{i}, & i=1,2, \\
\beta_{1}^{i}=1 \quad \text { on } \partial \mathcal{O}_{i}, & i=1,2, \\
\nabla \beta_{1}^{i} \neq 0 \text { in } \bar{\Omega}_{1} \backslash \mathcal{O}_{i}, & i=1,2,
\end{array}
$$

where $n$ stands for the unit exterior normal to $\Omega_{1}$ and $\mathcal{O}_{i}, i=1,2$.

Step 2: Let $\widetilde{B}_{i}$ and $B_{i}, i=1,2$ be balls such that $B_{1} \subset \subset \widetilde{B}_{1} \subset \subset \mathcal{O}_{1}$ and $B_{2} \subset \subset \widetilde{B}_{2} \subset \subset \mathcal{O}_{2}$. We will present only the construction of $\widetilde{\beta}_{1}^{1}$. The second function will be obtained by the same arguments. Let us set

$$
W_{\varepsilon}=\left\{x: x \in \mathcal{O}_{1}, \quad \operatorname{dist}\left(x, \partial \mathcal{O}_{1}\right)<\varepsilon\right\}
$$

First, we observe that since $\partial_{n} \beta_{1}^{1}>0$ on $\partial \mathcal{O}_{1}$ and $\beta_{1}^{1}=1$ on $\partial \mathcal{O}_{1}$, we construct a function $\beta_{1}^{1} \in C^{1}\left(\overline{W_{\varepsilon}}\right)$, such that there exists $\delta>0$ such that

$$
\beta_{1}^{1} \leq 1 \quad \text { in } W_{\varepsilon}, \quad 0<\beta_{1}^{1} \leq 1-4 \delta \quad \text { on } \partial W_{\varepsilon} \backslash \partial \mathcal{O}_{1} \quad \text { and } \quad \nabla \beta_{1}^{1} \neq 0 \text { in } \overline{W_{\varepsilon}}
$$


Then, we can extend this function by a function still called $\beta_{1}^{1} \in C^{1}\left(\overline{\mathcal{O}}_{1}\right)$, such that

$$
0<\beta_{1}^{1} \leq 1-3 \delta \text { in } \mathcal{O}_{1} \backslash W_{\varepsilon} \text { and } \nabla \beta_{1}^{1} \neq 0 \quad \text { in } \overline{W_{\varepsilon}} .
$$

Now, we approximate $\beta_{1}^{1}$ by Morse functions in such a way that

$$
0<\beta_{1}^{1} \leq 1-2 \delta \quad \text { in } \mathcal{O}_{1} \backslash W_{\varepsilon},
$$

where we keep the name $\beta_{1}^{1}$ for this approximation. The gradient of this function vanishes only in a finite number of points. As we already mentioned in the Step 3 of this proof, we can deduce the existence of a diffeomorphism on $\mathcal{O}_{1}$, which keeps invariant $\overline{W_{\varepsilon}}$ and transports the points where the gradient of $\beta_{1}^{1}$ vanishes in $B_{1}$. We obtain then a new function (that we keep on calling $\beta_{1}^{1}$ ) such that $\beta_{1}^{1} \in C^{1}\left(\bar{\Omega}_{1}\right), \beta_{1}^{1}>0$ in $\Omega_{1}$ and

$$
\nabla \beta_{1}^{1} \neq 0 \quad \text { in } \bar{\Omega}_{1} \backslash B_{1} .
$$

Moreover, from (217) we obtain that for $\delta>0$ we have

$$
\beta_{1}^{1} \leq 1-\delta \text { in } \bar{B}_{1} .
$$

Analogously, we construct a function $\beta_{1}^{2} \in C^{1}\left(\bar{\Omega}_{1}\right), \beta_{1}^{2}>0$ in $\Omega_{1}$, which verifies

$$
\nabla \beta_{1}^{2} \neq 0 \quad \text { in } \bar{\Omega}_{1} \backslash B_{2}
$$

and

$$
\beta_{1}^{2} \leq 1-\delta \quad \text { in } \bar{B}_{2}
$$

Step 3: Let us finally prove that the properties (40) and (41) are satisfied. For this, we will see that it is possible to modify $\beta_{1}^{1}$ (resp. $\beta_{1}^{2}$ ) in $B_{1}$ (resp. $B_{2}$ ) in order to obtain the conditions (40) and (40). We will be able to do this without changing the values of these functions in $\overline{\mathcal{O}}_{1} \backslash B_{1}$ and $\overline{\mathcal{O}}_{2} \backslash B_{2}$. For simplicity, we will present the details of the construction of only one of such a function, because the same arguments will be valid for the other one.

Let us define a new function $\widetilde{\beta}_{1}^{1}$ as follows:

$$
\widetilde{\beta}_{1}^{1}(x)=\left\{\begin{array}{lll}
\beta_{1}^{1}(x) & \text { if } & x \in \bar{\Omega}_{1} \backslash \mathcal{O}_{1}, \\
\left(\beta_{1}^{1}(x)\right)^{n(x)} & \text { if } & x \in \overline{\mathcal{O}}_{1},
\end{array}\right.
$$

with

$$
n(x)=\left(\frac{1}{\beta_{1}^{1}(x)}\right)^{p}
$$

where $p \in \mathbb{N}$ will be fixed later on. We can write that

$$
\widetilde{\beta}_{1}^{1}=\left(\beta_{1}^{1}\right)^{n}=\mathrm{e}^{n \log \beta_{1}^{1}} \quad \text { in } \overline{\mathcal{O}}_{1} .
$$

Since $\beta_{1}^{1}=1$ on $\partial \mathcal{O}_{1}$, we have that $n=1$ on $\partial \mathcal{O}_{1}$ and then, from (224) we deduce that

$$
\widetilde{\beta}_{1}^{1}=\beta_{1}^{1}=1 \quad \text { on } \partial \mathcal{O}_{1} .
$$


Moreover, we have that

$$
\nabla \widetilde{\beta}_{1}^{1}=\nabla \beta_{1}^{1} \quad \text { on } \partial \mathcal{O}_{1}
$$

and that the gradient of $\widetilde{\beta}_{1}^{1}$ vanishes only in $B_{1}$, where the gradient of $\beta_{1}^{1}$ is zero, i.e.

$$
\nabla \widetilde{\beta}_{1}^{1} \neq 0 \text { in } \bar{\Omega}_{1} \backslash B_{1}
$$

Indeed, using (224) and (223), we have

$$
\nabla \widetilde{\beta}_{1}^{1}=\left(\beta_{1}^{1}\right)^{n}\left(\nabla n \log \beta_{1}^{1}+\frac{n \nabla \beta_{1}^{1}}{\beta_{1}^{1}}\right)=\left(\beta_{1}^{1}\right)^{n} \nabla \beta_{1}^{1}\left[\frac{-p \log \beta_{1}^{1}}{\left(\beta_{1}^{1}\right)^{p+1}}+\frac{1}{\left(\beta_{1}^{1}\right)^{p+1}}\right] .
$$

Taking into account (217), it is easy to deduce from (228), that (226) and (227) hold.

In order to modify the values of $\widetilde{\beta}_{1}^{1}$ in $B_{1}$, we first use (219) and we obtain that

$$
n \geq\left(\frac{1}{1-\delta}\right)^{p}
$$

Next, from (224) we deduce that

$$
\widetilde{\beta}_{1}^{1} \leq(1-\delta)^{(1 /(1-\delta))^{p}}
$$

On the other hand, we know that the second function that we constructed in the Step 5 of this proof satisfies

$$
0<\beta_{1}^{2} \leq \bar{\beta}_{1}^{2}=\max _{\bar{B}_{1}} \beta_{1}^{2}>0 \quad \text { in } B_{1} .
$$

Choosing now $p$ large enough, we can deduce from the estimate (229) the following:

$$
\widetilde{\beta}_{1}^{1} \leq \frac{1}{2} \bar{\beta}_{1}^{2} \quad \text { in } B_{1}
$$

This gives (41). The same arguments applied to the function $\beta_{1}^{2}$ lead to the existence of a new function $\widetilde{\beta}_{1}^{2} \in C^{1}\left(\bar{\Omega}_{1}\right), \widetilde{\beta}_{1}^{2}>0$ in $\Omega_{1}$, such that satisfies $(38,40)$ and $(42)$ for $i=2$.

To conclude this step, we observe that we can approximate the functions of class $C^{1}$ already constructed by functions of class $C^{2}$, preserving the properties of the functions $\widetilde{\beta}_{1}^{1}$ and $\widetilde{\beta}_{1}^{2}$.

Step 4: For $\varepsilon, \varepsilon^{\prime}>0$ small enough, we set

$$
V_{\varepsilon}(\Gamma)=\left\{x: x \in \Omega_{0}, \quad \operatorname{dist}(x, \Gamma)<\varepsilon\right\}
$$

and

$$
V_{\varepsilon^{\prime}}(S)=\left\{x: x \in \Omega_{0}, \quad \operatorname{dist}(x, S)<\varepsilon^{\prime}\right\}
$$

We can locally construct a function $\alpha_{0}$ in $\overline{V_{\varepsilon}(\Gamma)}$, such that $\alpha_{0} \in C^{2}\left(\overline{V_{\varepsilon}(\Gamma)}\right)$ and

$$
\begin{array}{ll}
\alpha_{0}=0 & \text { on } \Gamma, \quad \partial_{n} \alpha_{0}>0 \quad \text { on } \Gamma \\
\alpha_{0}>0 & \text { in } \overline{V_{\varepsilon}(\Gamma)}, \nabla \alpha \neq 0 \quad \text { in } \overline{V_{\varepsilon}(\Gamma)} .
\end{array}
$$


On the other hand, in $\overline{V_{\varepsilon^{\prime}}(S)}$ we construct another function, which for simplicity, we also will denote by $\alpha_{0}$, such that $\alpha_{0} \in C^{2}\left(\overline{V_{\varepsilon^{\prime}}(S)}\right)$ and

$$
\begin{aligned}
& \alpha_{0}=2 \quad \text { on } S, \quad \partial_{n} \alpha_{0}>0 \quad \text { on } S, \\
& \alpha_{0}>0 \text { in } \overline{V_{\varepsilon^{\prime}}(S)}, \nabla \alpha_{0} \neq 0 \text { in } \overline{V_{\varepsilon^{\prime}}(S)}
\end{aligned}
$$

and

$$
a_{0} \partial_{n} \alpha_{0}=a_{1} \partial_{n} \beta_{1}^{i} \quad \text { on } S, \quad i=1,2 .
$$

Now, we extend both functions in $\Omega_{0}$, to a function which we keep on calling $\alpha_{0}$, with the following properties:

$$
\begin{aligned}
& \alpha_{0} \in C^{2}\left(\bar{\Omega}_{0}\right), \quad \alpha_{0}>0 \quad \text { in } \Omega_{0}, \\
& \alpha_{0}=0 \quad \text { on } \Gamma, \partial_{n} \alpha_{0}>0 \text { on } \Gamma, \\
& \alpha_{0}=2 \quad \text { on } S, \partial_{n} \alpha_{0}>0 \text { on } S .
\end{aligned}
$$

Step 5: In the sequel, we will use same arguments as for the proof of Lemma 3.1.

Thanks to the Morse theorem, we deduce that there exists a sequence of Morse functions $\theta_{k}, k \geq 1$ (functions with isolated critical points i.e. their gradient vanishes only in a finite number of points), such that

$$
\theta_{k} \rightarrow \alpha_{0} \quad \text { in } C^{2}\left(\bar{\Omega}_{0}\right) \quad \text { if } k \rightarrow+\infty .
$$

If $\theta_{k}$ is close enough to $\alpha_{0}$, the points where $\nabla \theta_{k}$ vanishes can not be in $\overline{V_{\varepsilon}(\Gamma)} \cup \overline{V_{\varepsilon^{\prime}}(S)}$. Moreover, we can assume that for some $\delta>0$ we have

$$
\left|\nabla \alpha_{0}\right| \geq \delta>0 \quad \text { in } \overline{V_{\varepsilon}(\Gamma)} \cup \overline{V_{\varepsilon^{\prime}}(S)} .
$$

We can construct a Morse function $\mu \in C^{2}\left(\bar{\Omega}_{0}\right)$, such that

$$
\begin{aligned}
& \mu=0 \quad \text { on } \Gamma, \quad \partial_{n} \mu<0 \quad \text { on } \Gamma \text {, } \\
& \mu=2 \quad \text { on } S, \quad \partial_{n} \mu>0 \quad \text { on } S
\end{aligned}
$$

and

$$
\nabla \mu \neq 0 \quad \text { in } \overline{V_{\varepsilon}(\Gamma)} \cup \overline{V_{\varepsilon^{\prime}}(S)} .
$$

Indeed, it suffices to consider $\varphi \in \mathcal{D}\left(\overline{V_{\varepsilon}(\Gamma)} \cup \overline{V_{\varepsilon^{\prime}}(S)}\right)$ and $\varphi=1$ in a neighborhood of $\Gamma \cup S$ and to define

$$
\mu_{k}(x)=\theta_{k}(x)+\varphi(x)\left(\alpha_{0}(x)-\theta_{k}(x)\right) .
$$

Arguing as in the proof of Lemma 3.1, we can choose $k \geq k_{0}$ and $\mu(x)=\mu_{k}(x)$ in such a way that $\mu$ is a Morse function with gradient vanishing only in the points contained in the set of points where the gradient of $\theta_{k}$ vanishes and satisfying the previous properties.

Step 6: Finally, we can deduce that there exists a mapping $g: \Omega \mapsto \Omega$ which is a diffeomorphism on $\Omega$, which leaves invariant $\overline{V_{\varepsilon}(\Gamma)} \cup \overline{V_{\varepsilon^{\prime}}(S)}$ and transports the points where the gradient of $\mu$ vanishes in $\omega_{0}$. We set

$$
\widetilde{\beta}_{0}(x)=\mu(g(x)) .
$$

This ends the proof of Lemma 3.2. 
We thank the referee for useful remarks and corrections concerning some technical points of this article.

\section{REFERENCES}

[1] S. Anita and V. Barbu, Null controllability of nonlinear convective heat equation. ESAIM: COCV 5 (2000) 157-173.

[2] D.G. Aronson and J. Serrin, Local behavior of solutions of quasilinear parabolic equations. Arch. Rational Mech. Anal. 25 (1967) 81-122.

[3] D.G. Aronson and J. Serrin, A maximum principle for nonlinear parabolic equations. Ann. Scuola Norm. Sup. Pisa 3 (1967) 291-305

[4] J.P. Aubin, L'analyse non linéaire et ses motivations économiques. Masson (1984).

[5] V. Barbu, Exact controllability of the superlinear heat equation. Appl. Math. Optim. 42 (2000) 73-89.

[6] T. Cazenave and A. Haraux, Introduction aux problèmes d'évolution semi-linéaires. Ellipses, Paris, Mathématiques 86 Applications (1990).

[7] S. Cox and E. Zuazua, The rate at which energy decays in a string damped at one end. Indiana Univ. Math. J. 44 (1995) 545-573.

[8] A. Doubova, E. Fernández-Cara, M. González-Burgos and E. Zuazua, On the controllability of parabolic system with a nonlinear term involving the state and the gradient. SIAM: SICON (to appear).

[9] C. Fabre, J.-P. Puel and E. Zuazua, (a) Approximate controllability for the semilinear heat equation. C. R. Acad. Sci. Paris Sér. I Math. 315 (1992) 807-812; (b) Approximate controllability of the semilinear heat equation. Proc. Roy. Soc. Edinburgh Sect. A 125 (1995) 31-61.

[10] C. Fabre, J.-P. Puel and E. Zuazua, Approximate controllability for the linear heat equation with controls of minimal $L^{\infty}$ norm. C. R. Acad. Sci. Paris Sér. I Math. 316 (1993) 679-684.

[11] E. Fernández-Cara, Null controllability of the semilinear heat equation. ESAIM: COCV 2 (1997) 87-107.

[12] E. Fernández-Cara and E. Zuazua, The cost of approximate controllability for heat equations: The linear case. Adv. Differential Equations 5 (2000) 465-514.

[13] E. Fernández-Cara and E. Zuazua, Null and approximate controllability for weakly blowing up semilinear heat equations. Ann. Inst. H. Poincaré Anal. Non Linéaire 17 (2000) 583-616.

[14] E. Fernández-Cara and E. Zuazua, On the null controllability of the one-dimensional heat equation with BV coefficients (to appear).

[15] A. Fursikov and O.Yu. Imanuvilov, Controllability of Evolution Equations. Seoul National University, Korea, Lecture Notes 34 (1996).

[16] O.Yu. Imanuvilov, Controllability of parabolic equations. Mat. Sb. 186 (1995) 102-132.

[17] O.Yu. Imanuvilov and M. Yamamoto, Carleman estimate for a parabolic equation in a Sobolev space of negative order and its applications. Lecture Notes in Pure Appl. Math. 218 (2001) 113-137.

[18] O.A. Ladyzenskaya, V.A. Solonnikov and N.N. Uraltzeva, Linear and Quasilinear Equations of Parabolic Type. Nauka, Moskow (1967).

[19] A. Pazy, Semigroups of linear operators and applications to partial differential equations. Springer-Verlag, New York (1983).

[20] D.L. Russell, A unified boundary controllability theory for hyperbolic and parabolic partial differential equations. Stud. Appl. Math. 52 (1973) 189-211.

[21] F.B. Weissler, Local existence and nonexistence for semilinear parabolic equations in $L^{p}$. Indiana Univ. Math. J. 29 (1980) $79-102$.

[22] F.B. Weissler, Semilinear evolution equations in Banach spaces. J. Funct. Anal. 32 (1979) 277-296.

[23] E. Zuazua, Exact boundary controllability for the semilinear wave equation, in Nonlinear Partial Differential Equations and their Applications, Vol. X, edited by H. Brezis and J.-L. Lions. Pitman (1991) 357-391.

[24] E. Zuazua, Finite dimensional controllability for the semilinear heat equations. J. Math. Pures 76 (1997) 570-594.

[25] E. Zuazua, Approximate controllability for semilinear heat equations with globally Lipschitz nonlinearities. Control and Cybernetics 28 (1999) 665-683. 